\title{
Topology optimization for minimum weight with compliance and simplified nominal stress constraints for fatigue resistance
}

\author{
Maxime Collet • Matteo Bruggi • Pierre Duysinx
}

Received: date / Accepted: date

\begin{abstract}
This work investigates a simplified approach to cope with the optimization of preliminary design of structures under local fatigue constraints along with a global enforcement on the overall compliance. The problem aims at the minimization of the weight of linear elastic structures under given loads and boundary conditions. The expected stiffness of the optimal structure is provided by the global constraint, whereas a set of local stress-based constraints ask for a structure to be fatigue resistant. A modified Goodman fatigue strength comparison is implemented through the same formalism to address pressure--dependent failure in materials as in Drucker-Prager strength criterion. As a simplification, the Sines approach is used to define the equivalent mean and alternating stresses to address the fatigue resistance for an infinite life time. Sines computation is based on the equivalent mean and alternate stress depending on the invariants of the stress tensor and its
\end{abstract}

Maxime Collet

Department of Aerospace and Mechanical Engineering University of Liège, 4000 Liège, Belgium

Tel.: +32-4-3669273

Fax: $+32-4-3669159$

E-mail: maxime.collet@ulg.ac.be

Matteo Bruggi

Department of Civil and Environmental Engineering

Politecnico di Milano, I20133, Milano, Italy

Tel.: +39-02-23994232

Fax: +39-02-23994220

E-mail: matteo.bruggi@polimi.it

Pierre Duysinx

Department of Aerospace and Mechanical Engineering

University of Liège, 4000 Liège, Belgium

Tel.: +32-4-3669194

Fax: +32-4-3669159

E-mail: p.duysinx@ulg.ac.be deviatoric part, respectively. The so-called singularity phenomenon is overcome by the implementation of a suitable $q p$-relaxation of the equivalent stress measures. Numerical examples are presented to illustrate the features of the achieved optimal layouts and of the proposed algorithm.

Keywords topology optimization · fatigue constraints · stress constraints · singularity problem · Goodman criterion

\section{Introduction}

Topology optimization is formulated as the problem that finds the optimal distribution of a given amount of material within a design domain, see Bendsøe and Sigmund (2003). Since the seminal work by Bendsøe and Kikuchi (1988), most developments of topology optimization have been based on the minimization of the elastic strain energy of the structure related to the external work of the loads. This problem is commonly named the minimization of the structural compliance or, in other words, the maximization of the overall stiffness. Today, aeronautical and mechanical industries consider topology optimization as a powerful tool for conceptual design in engineering. However in real life applications, a conventional compliancebased topology optimization can not fully satisfy the industrial needs. To produce structural parts that fulfil specific functioning requirements, failure of the structure must be prevented at each point of the component. Therefore, as pointed out in Duysinx et al (2008), a stress-based topology optimization is needed to propose a design that can satisfy given stress-based requirements. Fur- 
thermore, many aeronautical and automotive applications require that the life of a structure is preserved at least during a given amount of loading cycles. In this way, the structure must be designed such that it can sustain fatigue failure.

Compliance-based topology optimization is well mastered and there are many successful results, see for instance Eschenauer and Olhoff (2001), Rozvany (2009) and Bendsøe and Sigmund (2003) for a comprehensive review. Conversely, stress-based topology optimization was neglected, for a long time, mainly because of many related numerical issues. Since the last decades, a renewed interest has been directed towards this approach as we can seen from some milestone works, see Duysinx and Bendsøe (1998), Duysinx and Sigmund (1998), Svanberg et Werme (2007), Peirera et al (2004), Paris et al (2009) and Guilherme et Fontsesca (2007) for example. The main encountered issues in stressbased topology optimization are the singularity phenomenon and the large scale optimization problem due to the local behavior of the stresses.

The so-called singularity phenomenon was extensively studied by Sved and Ginos (1968) and Kirsch (1990), among the others. This phenomenon can lead to non convergence issues, meaning that, when the density approaches its lower bound, the stress value is different from zero and the minimization algorithm is steered towards local solutions full of gray regions. The singularity phenomenon can be overcome through an appropriate relaxation of the constraints in the optimization problem. The so-called $\varepsilon-$ relaxation was first proposed by Cheng and Guo (1997). This method resorts to the solution of a series of perturbed suboptimization problems with a decreasing value of a relaxing parameter $\varepsilon$. Later on, Bruggi (2008) proposed an alternative technique, the so-called $q p$ relaxation, which adopts a different penalization for the interpolation of stiffness $(p)$ and apparent "local" stresses $(q)$.

An additional numerical issue affecting a stressbased topology optimization is the large size of the optimization problem. It is well established that stress-based topology optimization leads to large scale problems when the control of the stress measure is operated at each point of the structure. That is to say that there are at least as many constraints as the design variables are. This approach, called the local stress approach, was originally proposed by Duysinx and Bendsøe (1998). It has been shown that the crucial point was the computational effort to solve the optimization prob- lem. A global stress approach, using aggregation of the local constraints techniques either based on the $p$-norm, the $p$-mean or the Kresselmeier Steinhauser (KS) function were proposed by Duysinx and Sigmund (1998) and Yang and Chen (1996) to overcome the issue related to the previous local approach. It was shown the the global CPU time was effectively reduced, but the robustness of a local control was generally lost. It must be also noticed that, due to the highly non linear behavior of such a global constraint, the resolution of the problem can become unstable and parameter-dependant. Luo et al (2013) proposed an enhanced aggregation technique based on the KS function which aggregates separately the active and the passive local stress constraints along with a relevant active set strategy. The exposed method showed interesting results but still requires a good tuning of the numerical parameters. Between the local and the global approach, Holmberg et al $(2013,2014)$ used clustered stress constraints. The method is based on the aggregation of the local stress constraints into clusters allowing for a decent control of the local peak value of the stresses. The classical global approach is recovered when only one cluster is used. The main issue when using the proposed clustering method lies in the way to generate clusters approximating suitably the local stresses. Furthermore, it has been shown that the solution depends on the number of clusters. An alternative to reduce the CPU time was proposed by Bruggi and Duysinx $(2012,2013)$ which consider a global compliance constraint along with local stress constraints and an enhanced active-set strategy. Results showed that the number of local stress constraints is highly reduced when stress peaks are dealt with and the global compliance enforcement is active. This leads to smaller CPU time even if the number of stress constraints remains higher than in a global or clustered approach.

When dealing with stress-based topology optimization, the possibility to take into account fatigue within the optimization procedure is a natural extension of the available developments. Fatigue is one of the most critical mode of failure in many engineering applications such as aeronautical and automotive systems.. Today, transports applications seek for lighter and optimal design with respect to environmental care and conservation. Despite these obvious industrial needs, as pointed out in Holmberg et al (2014), there is not a wide literature about fatigue failure in topology optimization, although some interesting issues were 
explored concerning shape optimization, see e.g. Mrzygold and Zielinski (2006), Kaya et al (2010), Grunwald and Schnack (1997). The work by Holmberg et al (2014) introduces probability-based fatigue constraints, which are aggregated through the adoption of stress clusters. The contribution in Svärd (2015) deals with fatigue introducing an assumption on the failure probability of a volume element. It is based on the idea that the weakest link model of failure developed by Weibull (1939) takes a $p$-norm like form that is suitable for stressbased topology optimization. The work in Seung et al (2015) proposes another approach by incorporating dynamic fatigue and static failure criterion under constant and proportional mechanical loads. Aggregation methods are used to tackle local fatigue constraints, whereas a differentiable form of the signed von Mises criterion is implemented to detect compressive and tensile stress states within the design when defining the mean component of the stress. Sherif et al (2010) used the concept of the equivalent static load (ESL) in topology optimization as shown in Park and Kang (2003) and Kim and Park (2010) to sustain fatigue. This approach uses a given tolerated damage as endcriterion and looks after a design that fulfils the constraints on the damage by performing several FEM calculations coupled to a topology optimization code. The main advantage of the proposed procedure is that it can solve large scale problems. However, a full dynamic analysis is required to generate the ESL, as well as fatigue calculation to compute the most critical damage linked to the relevant ESL.

Within the above framework, the present work provides a simplified method that can improve conventional compliance-based layouts with respect to fatigue, adopting the mechanical design approach of machine elements based on $S-N$ diagrams or fatigue criteria. This can be done resorting to an extension of the formulation proposed in Bruggi and Duysinx (2012) for stress-constrained topology optimization of stiff structures with non-symmetric strength in tension and compression. Indeed, the Sines method, that is used to define an equivalent stress measure for both the mean and alternating component, respectively, is based on the first and second invariants of the stress tensor, i.e hydrostatic and deviatoric. To steer fatigue resistance, a modified Goodman fatigue criterion is implemented through the same formalism originally adopted in Bruggi and Duysinx (2012) to address pressure-dependent failure through the smooth
Drucker - Prager criterion. In the proposed formulation, the compliance constraint deals with the serviceability of the structure, enforcing a limit on the displacement of the loaded point, whereas selected active local constraints steer the optimizer towards the achievement of fatigue-resistant layouts, as required by industrial applications. The proposed procedure is particularly effective when limited regions of the design govern the collapse of the structure.

The paper is organized as follows. Section 2 presents the Goodman failure criterion based on the Sines method, along with the related equivalent stress measures to be used in conjunction with the SIMP material. The computation of the stresses and fatigue criteria in the finite elements formalism used in this work, i.e. a displacementbased approximation, is also illustrated. Section3.1 presents the formulation of the topology optimization problem which aims at the minimization of the structural weight with compliance and fatigue constraints. Section 3.3 mentions some numerical issues, such as the adopted selection strategy for stress constraints, the mathematical relaxation to overcome the singularity problem and the limitations experienced by conventional formulations of topology optimization when coping with the approximation of the stress field. The sensitivity computation is performed through the adjoint method, as it is dealt with in Section 3.2. Section 4 presents numerical results achieved through the above procedure, providing comparisons with optimal layouts achieved by stress-based topology optimization based the von Mises stress and fatigue stress constraints. Section 5 concludes the paper, formulating remarks and drawing limitations of the proposed preliminary design approach.

\section{The fatigue failure criterion}

This section aims at presenting the approach followed in this paper to handle fatigue within a topology optimization problem. The adopted procedure follows the stress-based approach that is used for fatigue design in machine design, see e.g. Budynas and Nisbett (2011) and Norton (2000). This means that the presented framework is valid only in the high-cycle regime, i.e. for a very large number of cycles (typically $\geq 10^{3}$ although there is no real consensus about the threshold between Low Cycle Fatigue and High Cycle Fatigue). In the following, it is assumed that we are in the case of High Cycle Fatigue (HCF). It is important to pinpoint the 
fact that HFC modeling works well when the loads are known and predictable, meaning not random. Furthermore, this approach is consistent as long as the material remains elastic and no crack initiation is found. Finally, in absence of corrosion, at room temperature and for a constant loading, the quiescence of the solicitation has no influence on the life of the structure and one has therefore to only consider the amplitude, see Norton (2000). In those conditions, the design can be performed through a equivalent static analysis as discussed in Section 2.2.

\subsection{Sines method and modified Goodman criterion}

Following the adopted assumptions of the former paragraph, it is a general knowledge in machine design theory that a loading condition can be modeled as shown in Figure 1. A cycle consists of two reversals of the stress state $(\sigma)$ over time $(t)$. An alternate part $\left(\sigma_{a}\right)$ and a mean part $\left(\sigma_{m}\right)$ of the stress measure can be defined thanks to its maximum $\left(\sigma_{\max }\right)$ and minimum $\left(\sigma_{\min }\right)$ values as:

$$
\left\{\begin{array}{l}
\sigma_{a}=\frac{\sigma_{\max }-\sigma_{\min }}{2} \\
\sigma_{m}=\frac{\sigma_{\max }+\sigma_{\min }}{2}
\end{array} .\right.
$$

In the early studies on fatigue failure, as in the work of August Whöler reported in Schütz (1996), only the alternate component was considered leading to the well-known Whöler curve. As taught by many originally unpredicted collapses, structures do not experience only fully-reversed loadings as considered in the Wölher curve but are also submitted to constant components, here and now referred to mean component. A new diagram, the so-called Haigh diagram was developed to account both for the alternate part $\sigma_{a}$ and for the mean part $\sigma_{m}$ of the stress, see Lalanne (1999).

The approach followed in this paper is based on the one adopted in design of machine elements, see e.g Norton (2000) and Budynas and Nisbett (2011) for details. The design resorts to a static analysis and the fatigue resistance is ruled by the (simplified) "Augmented modified Goodman diagram".

The analysis reported in Section 4 assume that the fatigue resistance is predicted by the Goodman diagram represented in Figure 2. It refers to infinite life of the structure, meaning that the specimen can sustain more than $10^{7}$ cycles without breaking. As explained in the following, the alternating component is bounded by through an endurance limit $S_{e}$ corresponding to the value of the allowable stress for an infinite number of cycles. Alternatively, a higher value of $S_{e}$ could be adopted for a given lower number of cycles.

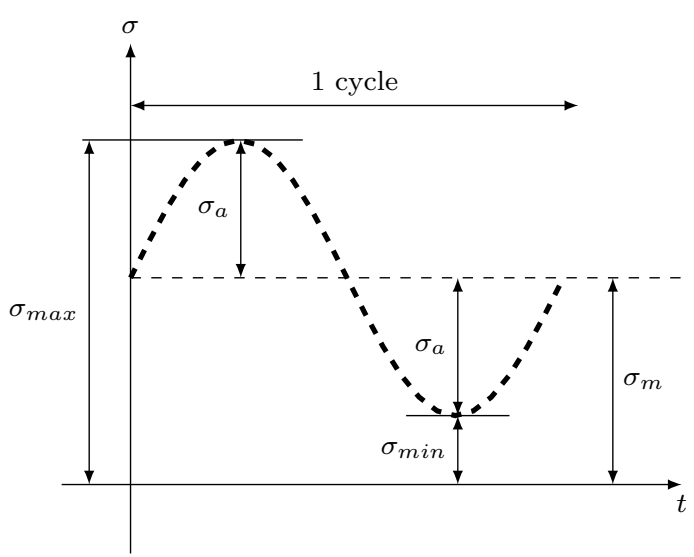

Fig. 1 One cycle loading

The computational procedure adopted in this work can be summarized as follows:

- compute an equivalent measure of the alternating and mean stress ( $\sigma_{a}^{e q}$ and $\sigma_{m}^{e q}$, respectively) within the structure, based on a static analysis;

- compare the stress state obtained with the Goodman diagram.

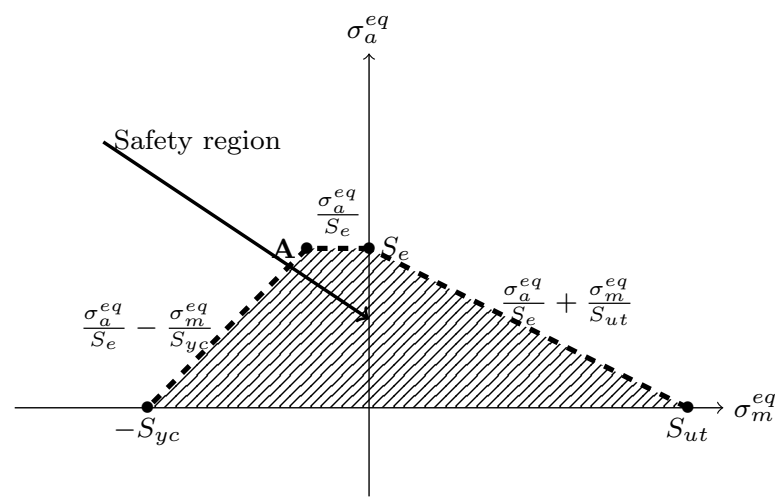

Fig. 2 (Simplified) Augmented modified Goodman diagram

The line connecting point $\left(-S_{y c}, 0\right)$ to point 'A' defines yielding in compression. The line connecting point 'A' to $S_{e}$ restricts the stress state in case of compressive mean stress, meaning that any beneficial effect due to $\sigma_{m}^{e q}<0$ is neglected to 
make the criterion more conservative. Finally, the straight line connecting point $\left(0, S_{e}\right)$ to $\left(S_{u t}, 0\right)$ defines fatigue failure in case of tensile mean stress

Usually in fatigue design some intensity factors are needed to compute the actual stresses in highly stresses regions, thus accounting for geometric singularities such as notches. As in topology optimization designs, the whole layout is determined with no a priori assumption on the shape of the optimal structure. Hence, none of these factors will be adopted within the proposed framework, which aims at providing a preliminary optimal solution to help the designer in the achievement of stiff fatigue-resistant structural components. Further remarks on the limitation of the proposed approach related to the accuracy in the approximation of the stress field are reported in Section 3.3.

Due to the adopted linear elastic modeling, the component of the stress tensor $\sigma_{i j}$ can be written as the sum of an alternate $\sigma_{a, i j}$ and mean part $\sigma_{m, i j}$, see Figure 1, as:

$\sigma_{i j}=\sigma_{a, i j}+\sigma_{m, i j}=c_{a} \cdot \sigma_{i j}+c_{m} \cdot \sigma_{i j}$.

In the above equation, $c_{a}$ and $c_{m}$ are user-defined parameters that allow scaling $\sigma_{i j}$ to $\sigma_{a, i j}$ and $\sigma_{m, i j}$ respectively, being $c_{a}+c_{m}=1$.

For $2 \mathrm{D}$ and $3 \mathrm{D}$ stress state, according to Sines theory, see Norton (2000), the equivalent stress measures accounting for the alternate and the mean parts of the stress tensor, $\sigma_{a}^{e q}$ and $\sigma_{m}^{e q}$ respectively, can be written using the second and the first invariant of this these tensors:

$\left\{\begin{array}{l}\sigma_{a}^{e q}=\sqrt{3 J_{2 D}\left(\sigma_{a, i j}\right)} \\ \sigma_{m}^{e q}=J_{1}\left(\sigma_{m, i j}\right)\end{array}\right.$,

where $J_{2 D}\left(\sigma_{a, i j}\right)$ is the second deviatoric stress invariant computed from the alternate part $\sigma_{a, i j}$, whereas $J_{1}\left(\sigma_{m, i j}\right)$ is the first stress invariant of the mean part $\sigma_{m, i j}$. Under the assumption of plane stress conditions one has:

$$
\left\{\begin{array}{l}
3 J_{2 D}\left(\sigma_{a, i j}\right)=\sigma_{a, 11}^{2}+\sigma_{a, 22}^{2}-\sigma_{a, 11} \sigma_{a, 22}+3 \sigma_{a, 12}^{2} \\
J_{1}\left(\sigma_{m, i j}\right)=\sigma_{m, 11}+\sigma_{m, 22}
\end{array}\right.
$$

It is worth noticing that Eqns. (2) and (3) have analytic forms that are very similar to those used in Bruggi and Duysinx (2012) to handle the DruckerPrager equivalent stress and address the optimal design of structures having a non-symmetric behavior in tension and compression. Within the proposed approach, a few modifications are needed to turn stress-constrained codes from strengthbased problems to fatigue--based optimal design. In addition, no problem of differentiability arises when detecting compressive and tensile values of the mean stress component, contrary to the situation encountered and solved by Seung et al (2015) with the signed von Mises criterion.

The safe region in the Goodman fatigue domain represented in Figure 2 can be defined by the following set of three inequalities:

$$
\left\{\begin{array}{l}
\frac{\sigma_{a}^{e q}}{S_{e}}+\frac{\sigma_{m}^{e q}}{S_{u t}} \leq 1 \\
\frac{\sigma_{a}^{e q}}{S_{y}}-\frac{\sigma_{m}^{e q}}{S_{y c}} \leq 1, \\
\frac{\sigma_{a}^{e q}}{S_{e}} \leq 1
\end{array},\right.
$$

where $S_{y}$ is the yielding stress in tension, $S_{y c}$ is the yielding stress in compression, $S_{e}$ is the endurance limit, and $S_{u t}$ is the ultimate stress. The set of inequalities of Eqn. (5) can be easily implemented within a stress-based formulation to handle the simplified version of the Goodman diagram represented in Figure 2.

Finally, note that the condition $\sigma_{a}^{e q} \geq 0$ (restricting the Goodman diagram in the first and second quadrants of the plane $\sigma_{m}^{e q}-\sigma_{a}^{e q}$ ) straightforwardly arises from the definition of the alternate equivalent stress in the Sines method, see statement in Eqn. (3-1).

\subsection{Implementation within a standard displacement-based code}

The well-known SIMP law, see e.g. Bendsøe and Sigmund (2003), is used to interpolate the elasticity tensor $C_{i j h k}(\rho(x))$ as a function of the values of the density $\rho$ :

$C_{i j h k}(\rho)=\rho^{p} C_{i j h k}^{0}$,

where $C_{i j h k}^{0}$ is the stiffness tensor for a given isotropic medium and $p=3$ is the penalization parameter herein adopted, as suggested in e.g. Bendsøe and Sigmund (2003).

A conventional displacement-based finite element method is adopted to solve equilibrium equations: four-node squared elements with bi-linear displacement shape functions are used, along with a piecewise constant discretization of the density field.

Recalling that the components of the stress tensor $\sigma_{i j}$ can be derived from the components of 
strain tensor $\varepsilon_{h k}$ at equilibrium. Eqn. (6) implies that $\sigma_{i j}=\rho^{p} C_{i j h k}^{0} \varepsilon_{h k}$. Focusing on the $e$-th finite element, the stress in a relevant point (herein the centroid) can be written in matrix form as $\sigma_{e}=x_{e}^{p} \mathbf{T}_{e}^{0} \mathbf{U}_{\mathbf{e}}$, being $\mathbf{U}_{\mathbf{e}}$ the displacement vector, $\mathbf{T}_{e}^{0}$ the stress matrix of the element $e$ made of virgin material and $x_{e}$ the $e$-th component of the vector of the $N$ element densities $\mathbf{x}$, see e.g. Duysinx and Sigmund (1998).

Taking advantage of the so-called "von Mises stress matrix" $\mathbf{M}_{e}^{01}$ and "hydrostatic stress matrix" $\mathbf{H}_{e}^{02}$, Eqn. (4) may be re-written as:

$$
\left\{\begin{array}{l}
3 J_{2 D, e}\left(\sigma_{i j}\right)=x_{e}^{2 p} \mathbf{U}_{e}^{T} \mathbf{M}_{e}^{0} \mathbf{U}_{e} \\
J_{1, e}\left(\sigma_{i j}\right)=x_{e}^{p} \mathbf{H}_{e}^{0} \mathbf{U}_{e}
\end{array},\right.
$$

meaning that the equivalent Sines stress measures for the $e$-th finite element read:

$\left\{\begin{array}{l}\sigma_{a}^{e q}=x_{e}^{p}\left(c_{a} \sqrt{\mathbf{U}_{e}^{T} \mathbf{M}_{e}^{0} \mathbf{U}_{e}}\right)=x_{e}^{p} \bar{\sigma}_{a, e}^{e q} \\ \sigma_{m}^{e q}=x_{e}^{p}\left(c_{m} \mathbf{H}_{e}^{0} \mathbf{U}_{e}\right)=x_{e}^{p} \bar{\sigma}_{m, e}^{e q}\end{array}\right.$

To account for an appropriate failure criterion involving the porous SIMP material, one should introduce the so-called apparent "local" stress $\left\langle\sigma_{i j}\right\rangle$ that may be assumed as $\left\langle\sigma_{i j}\right\rangle=\sigma_{i j} / x_{e}^{q}$, with $q>$ 1. Finally, a suitable form of the Goodman fatigue criterion in porous media to be used in the $e^{- \text {th }}$ finite element reads:

$$
\left\{\begin{aligned}
\frac{\left\langle\sigma_{a, e}^{e q}\right\rangle}{S_{e}}+\frac{\left\langle\sigma_{m, e}^{e q}\right\rangle}{S_{u t}} & =x_{e}^{(p-q)}\left(\frac{\bar{\sigma}_{a, e}^{e q}}{S_{e}}+\frac{\bar{\sigma}_{m, e}^{e q}}{S_{u t}}\right) \leq 1 \\
\frac{\left\langle\sigma_{a, e}^{e q}\right\rangle}{S_{y}}-\frac{\left\langle\sigma_{m, e}^{e q}\right\rangle}{S_{y c}} & =x_{e}^{(p-q)}\left(\frac{\bar{\sigma}_{a, e}^{e q}}{S_{y}}-\frac{\bar{\sigma}_{m, e}^{e q}}{S_{y c}}\right) \leq 1 . \\
\frac{\left\langle\sigma_{a, e}^{e q}\right\rangle}{S_{e}} & =x_{e}^{(p-q)} \frac{\bar{\sigma}_{a, e}^{e q}}{S_{e}} \leq 1
\end{aligned}\right.
$$

The endurance limit $S_{e}$ is determined experimentally on test of specimens with given size, shape, surface finish for which fatigue tests are performed in a controlled atmosphere (temperature, humidity, loading operation, etc). Indeed, a actual endurance limit $\overline{S_{e}}$ should be adopted by applying correction factors as explained in Norton (2000):

$\overline{S_{e}}=C_{\text {load }} \cdot C_{\text {size }} \cdot C_{\text {surf }} \cdot C_{\text {temp }} \cdot C_{\text {rel }} \cdot S_{e}$.

In the above equation, $C_{\text {load }}<1$ is set in case of a non-bending loading (e.g. for axial loading, as

$$
\begin{aligned}
{ }^{1} \mathbf{M}_{e}^{0} & =\mathbf{T}_{\mathbf{e}}^{\mathbf{0}, \mathbf{T}} \mathbf{V} \mathbf{T}_{\mathbf{e}}^{\mathbf{0}} \text { with } \mathbf{V}=\left(\begin{array}{ccc}
1 & -1 / 2 & 0 \\
-1 / 2 & 1 & 0 \\
0 & 0 & 3
\end{array}\right) \\
{ }^{2} \mathbf{H}_{e}^{0} & =\left(\begin{array}{l}
1 \\
1 \\
0
\end{array}\right) \mathbf{T}_{\mathbf{e}}^{\mathbf{0}}
\end{aligned}
$$

prevalent in a truss-like optimal design), $C_{\text {size }}$ concerns a structure whose size is not the same than the samples, $C_{\text {surf }}$ allows considering the surface finish, $C_{\text {temp }}$ gives information about the service temperature and $C_{\text {rel }}$ takes into account the fact that published data are average values. As stated above, the proposed simplified procedure has the aim of investigating preliminary layouts for enforcing fatigue-resistance of the component in first stage of the design process. For this reason the simplification $\overline{S_{e}}=S_{e}$ will be operated. The endurance limit is not an easy material parameter to determine. According to Norton (2000) we can set $S_{e}=0,5 S_{u t}$ for steels exhibiting $S_{u t}<200 \mathrm{ksi}$ (1379 MPa).

\section{The topology optimization problem}

\subsection{Problem formulation}

The minimization of the structural weight of a linear elastic structure under fatigue constraints and a global compliance constraint is dealt with. The discrete formulation of the optimization problem reads:

$$
\begin{cases}\min _{x_{m i n} \leq x_{e} \leq 1} & \mathcal{W}=\sum_{N} x_{e} V_{e} \\ \text { s.t. } \quad & \mathbf{K}(\mathbf{x}) \mathbf{U}=\mathbf{F}, \\ & \mathcal{C} / \mathcal{C}_{L} \leq 1, \\ & x_{e}^{(p-q)}\left(\frac{\bar{\sigma}_{a, e}^{e q}}{S_{e}}+\frac{\bar{\sigma}_{m, e}^{e q}}{S_{u t}}\right) \leq 1, \forall e \\ & x_{e}^{(p-q)}\left(\frac{\bar{\sigma}_{a, e}^{e q}}{S_{y}}-\frac{\bar{\sigma}_{m, e}^{e q}}{S_{y c}}\right) \leq 1, \forall e \\ & x_{e}^{(p-q)} \frac{\bar{\sigma}_{a, e}^{e q}}{S_{e}} \leq 1, \forall e\end{cases}
$$

The total weight $\mathcal{W}$ of the structure is computed multiplying the element density of each one of the $\mathrm{N}$ elements in the mesh, $x_{e}$, by the relevant volume, $V_{e}$. Eqn. (11-2) enforces the discrete equilibrium in terms of the global stiffness matrix $\mathbf{K}$, the global displacement vector $\mathbf{U}$ and the vector of external loads F. A global compliance constraint is introduced in Eqn. (11-3), enforcing an upper bound for the structural compliance $\mathcal{C}=\mathbf{U}^{T} \mathbf{K} \mathbf{U}$. $\mathcal{C}$ is twice the work of the external loads against the corresponding displacements at equilibrium, meaning that this constraint could be equivalently expressed in terms of the maximum displacement 
allowed in the loaded region. In the following numerical simulations, $\mathcal{C}_{L}=\alpha_{\mathcal{C}} \mathcal{C}_{0}$, where $\mathcal{C}_{0}$ is the compliance evaluated for the virgin material over the full domain and $\alpha_{\mathcal{C}}$ is a prescribed parameter. Eqns. (11-4,5,6) refer to the set of $3 \cdot N$ local stress constraints on the equivalent Goodman/Sines stress measures reported in Eqn. (9).

The problem in Eqn. (11) defines a minimum weight formulation with compliance and fatigue constraints, i.e. the MWCF problem. This formulation may be exploited when safety against fatigue failure is required along with a prescribed stiffness at the serviceability limit state. This improves conventional compliance-based layouts. Implementation of the MWCF formulation follows straightforwardly that of the minimum weight formulation with compliance and stress constraints, i.e. the MWCS problem investigated in Bruggi and Duys$\operatorname{inx}(2012)$.

It must be remarked that the adopted discretization of the displacement and density field is affected by the well-known checkerboard problem, see e.g.Sigmund and Petersson (1998). Moreover, a control of the thickness of the members resulting from the optimization procedure is needed to avoid mesh dependence. This work adopts the densitybased approach, see e.g. Bourdin (2001) and Bruns and Tortorelli (2001). The consistent filter used in this contribution follows the approach proposed in the work byLe et al. (2010) where the original design variables $x_{e}$ are mapped into a new set of physical unknown $\tilde{x}_{e}$ :

$$
\begin{aligned}
& \tilde{x}_{e}=\frac{1}{\sum_{N} H_{e i}} \sum_{N} H_{e i} x_{i}, \\
& H_{e i}=\sum_{N} \max \left(0, r_{\text {min }}-\operatorname{dist}(e, i)\right),
\end{aligned}
$$

where $\operatorname{dist}(e, i)$ is the distance between the centroid of the $e$-th and $i$-th element, whereas $r_{\text {min }}>$ $d_{m}$ is the filter radius, and $d_{m}$ is the reference length of the element edges. In the simulations presented next, $r_{\min }=1,5 d_{m}$ is assumed.

\subsection{Sensitivity analysis}

This section deals with the sensitivity computation for the local constraints in Eqn. $(11-4,5,6))$, as required at each iteration of the optimization procedure. The derivative of the global compliance constraint $\mathcal{C}$ with respect to the density design variables is herein omitted since it is classic result for the sake of brevity, see e.g. Bendsøe and Sigmund (2003).
The derivatives of the equivalent "local" measures of the alternate and mean stress for the $e$-th finite element, respectively $\sigma_{a, e}^{e q}$ and $\sigma_{m, e}^{e q}$, with respect to the density unknown $x_{k}$ can be formulated as follows:

$$
\begin{aligned}
\frac{\partial \sigma_{a, e}^{e q}}{\partial x_{k}} & =\delta_{e k}(p-q) x_{e}^{p-q-1} \bar{\sigma}_{a, e}^{e q}+\frac{\partial \bar{\sigma}_{a, e}^{e q}}{\partial x_{k}} x_{e}^{p-q} \\
\frac{\partial \sigma_{m, e}^{e q}}{\partial x_{k}} & =\delta_{e k}(p-q) x_{e}^{p-q-1} \bar{\sigma}_{m, e}^{e q}+\frac{\partial \bar{\sigma}_{m, e}^{e q}}{\partial x_{k}} x_{e}^{p-q} .
\end{aligned}
$$

The above equation requires the computation of the sensitivity of $\bar{\sigma}_{a, e}^{e q}$ and $\bar{\sigma}_{m, e}^{e q}$, which is caried out through the adjoint method. Reminding Eqn. (8), one has:

$$
\begin{aligned}
& \frac{\partial \bar{\sigma}_{a, e}^{e q}}{\partial x_{k}}=\lambda^{T} \frac{\partial \mathbf{K}}{\partial x_{k}} \mathbf{U}, \quad \text { where } \\
& \mathbf{K} \lambda=-\left[c_{a}\left(\mathbf{U}^{T} \mathbf{M}_{e}^{0} \mathbf{U}\right)^{-\frac{1}{2}} \mathbf{M}_{e}^{0} \mathbf{U}\right]^{T},
\end{aligned}
$$

and

$$
\begin{aligned}
& \frac{\partial \bar{\sigma}_{m, e}^{e q}}{\partial x_{k}}=\mu^{T} \frac{\partial \mathbf{K}}{\partial x_{k}} \mathbf{U}, \quad \text { where } \\
& \mathbf{K} \mu=-\left[c_{m} \mathbf{H}_{e}^{0}\right]^{T} .
\end{aligned}
$$

In the above forms, $\lambda$ and $\mu$ are the adjoint vectors computed via the solution of each adjoint problem of Eqns. (14) and (15). The solution of one additional (pseudo)-load case for the linear system in Eqn. (11-2) is required per each (active) constraint.

As done in Bruggi and Duysinx (2012), the stiffness matrix $\mathbf{K}$ is factorized at each iteration before evaluating the constraints and their sensitivities in order to speed up the computations.

\subsection{Numerical issues and remarks}

The optimization problem in Eqn. (11) is solved by resorting mathematical programming approach, adopting the Method of Moving Asymptotes by Svanberg (1987).

When handling stress-based topology optimization, a main issue is the so-called singularity phenomenon, see e.g Cheng and Guo (1997) and Kirsch (1990). Following Duysinx and Bendsøe (1998), the same SIMP penalization $p=q$ should ultimately be assumed for both stiffness and "local" stress interpolation. Due to the asymptotic behavior of the apparent "local" stress in Eqn. (9), degenerated sub-domains appear in the feasible domain and the optimizer based on KKT conditions 
is likely to be stuck in a false optimum with massive grey regions, instead of the expected $0-1$ design. A classical way to overcome this issue consists in relaxing stress constraints. According to the $q p$-approach proposed by Bruggi (2008), an exponent $q<p$ is adopted in the simulations to provide a strong relaxation in the regions of low density, without introducing any bias at full density. In the first iterations $q=2.6$ is adopted to highly relax the problem and encourage the activation of the global compliance constraint speeding up the optimization solution. Afterwards the value of the relaxing parameter $q$ is gradually increased to $q=2.75$. Indeed, preliminary numerical tests have shown that the adaptive version of this relaxation leads to a more efficient solution.

A lower bound $x_{\min }>0$ is needed for each density design variable $x_{e}$ to avoid singularity of the global stiffness matrix when solving Eqn. (11-2). The assumption $x_{\text {min }}=10^{-3}$ has been adopted in Section 4 to provide numerical results, as recommended Bendsøe and Sigmund (2003). Numerical tests have shown that the adopted implementation of the relaxed stress-constrained formulation in Eqn. (11) is not sensitive with respect to a variation of $x_{\min }$ in the range $10^{-3}-10^{-5}$.

Active restriction has been implemented to reduce the computational effort in terms of sensitivity computation and MMA optimization. Sets of active constraints with dimension $N_{a}$ are processed during the optimization instead of the full set of $3 \cdot N$ local constraints. Only the local stress constraints whose value is larger than 0,65 are considered as potentially active during the first iteration. This threshold is progressively increased in 10 steps and constantly set to 0,85 thereafter, see in particular Bruggi and Duysinx (2013). The number of active constraints changes during the optimization, thus introducing discontinuities in the multi-constrained minimization. Within the proposed MWCF formulation, the global compliance constraint has a crucial role in smoothing any discontinuity arising during the optimization because of the adopted selection strategy, see further comments in Section 4.2.

A final but crucial remark addresses the lack of accuracy affecting the approximation of the stress field in discretized problems of topology optimization. The CPU cost drastically limits the refinement of the finite elements mesh, meaning that, quite often, the adopted finite elements approximation does not succeed in predicting with high accuracy the exact value of a stress concentra- tion, especially in case of local overstressings due to geometrical details. Conventional schemes are generally able to detect at least the location of a stress concentration, thus leading to optimal layouts whose shape carefully avoid stress concentration and exhibit the general stress flows. On this point, reference is made in particular to the comprehensive review by Le et al. (2010) which addresses several numerical experiments reported in the literature for the stress-based design of the $\mathrm{L}_{-}$ shaped cantilever.

As extensively pointed out in Svärd (2015), numerical inaccuracy in the evaluation of the stress field is also due to the jagged nature of the optimal structure arising in the fixed finite element mesh used to handle the design domain. At the boundaries of the optimal layout, the transition from full material 1 to void material $x_{\text {min }}$ through the grey region due to the filter, see Eqn. (12), may result in an unexpected oscillation of the stresses over the jagged boundary following the mesh, thus affecting the stress-based optimal design. To solve this problem, Svärd (2015) proposes the adoption of a combined approach that consists of an interior value extrapolation for the stresses across the boundary. Effective results are shown adopting this approach which is not implemented here but could be conveniently implemented within the proposed approach to improve the accuracy of the method in future works.

\section{Numerical simulations}

The following examples focus on compliance-constrained fatigue-based topology optimization (MWCF problems) versus compliance-constrained strengthbased topology optimization using the von Mises criterion (MWCS problem), see in particular Bruggi and Duysinx (2012). Geometry and boundary conditions for the numerical applications are represented in Figure 3, where black zones are nondesign regions surrounding the applied point loads to avoid the overstress due to singularities in the application of the load. The number of finite elements for each discretization is shown in Table 1.

In case of fatigue-based problems, three combinations of the weighting factors in Eqn. (2), $c_{a}$ and $c_{m}$, are explored to investigate the sensitivity of the designs with respect to the ratio between the alternate component and respect to the mean stress, see Table 1.

A reference material with Young modulus $E=$ $1 N / m^{2}$ and Poisson's ratio $\nu=0,3$ is considered 


\begin{tabular}{llllllll}
\hline Example & Figure & Problem & $\mathrm{N}$ & $\mathcal{W} / \mathcal{W}_{0}$ & $\alpha_{c}=\mathcal{C} / \mathcal{C}_{0}$ & $\mathrm{CPU}$ & $N_{a}^{f}$ \\
\hline 1 & Figure 4(a) & $\mathrm{MWCS}_{1}$ & 10240 & 0,4163 & 2 & 967,9 & 26 \\
1 & Figure 4(b) & $\mathrm{MWCF}_{1}\left(c_{a}=0,7 ; c_{m}=0,3\right)$ & 10240 & 0,4257 & 2 & 4746 & 95 \\
1 & Figure 4(c) & $\mathrm{MWCF}_{2}\left(c_{a}=0,5 ; c_{m}=0,5\right)$ & 10240 & 0,4286 & 2 & 1163 & 46 \\
1 & Figure 4(d) & $\mathrm{MWCF}_{3}\left(c_{a}=0,3 ; c_{m}=0,7\right)$ & 10240 & 0,4257 & 2 & 614 & 34 \\
2 & Figure 7(a) & $\mathrm{MWCS}$ & 4096 & 0,3965 & 2 & 970,1 & 60 \\
2 & Figure 7(b) & $\mathrm{MWCF}_{1}\left(c_{a}=0,7 ; c_{m}=0,3\right)$ & 4096 & 0,437 & 2 & 1261 & 263 \\
2 & Figure 7(c) & $\mathrm{MWCF}_{2}\left(c_{a}=0,5 ; c_{m}=0,5\right)$ & 4096 & 0,4117 & 2 & 881 & 118 \\
2 & Figure 7(d) & $\mathrm{MWCF}_{3}\left(c_{a}=0,3 ; c_{m}=0,7\right)$ & 4096 & 0,3974 & 2 & 316,7 & 44 \\
3 & Figure 14(a) & $\mathrm{MWCS}_{1}\left(c_{a}=0,7 ; c_{m}=0,3\right)$ & 2560 & 0,4916 & 2 & 41,93 & 33 \\
3 & Figure 14(b) & $\mathrm{MWCF}_{1}$ & 0,5153 & 2 & 188,2 & 98 \\
3 & Figure 14(c) & $\mathrm{MWCF}_{2}\left(c_{a}=0,5 ; c_{m}=0,5\right)$ & 2560 & 0,5067 & 2 & 126,8 & 56 \\
3 & Figure 14(d) & $\mathrm{MWCF}_{3}\left(c_{a}=0,3 ; c_{m}=0,7\right)$ & 2560 & 0,5012 & 2 & 51,37 & 43 \\
\hline
\end{tabular}

Table 1 Summary of the optimization problems regarding the number of elements $(N)$, the weight ratio $\left(\mathcal{W} / \mathcal{W}_{0}\right)$ after optimization, the final non-dimensional compliance $\left(\mathcal{C} / \mathcal{C}_{0}\right)$, the number of active constraints at convergence $\left(N_{a}^{f}\right)$ and the overall CPU time.

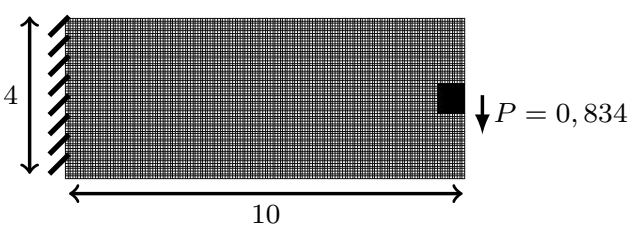

(a)

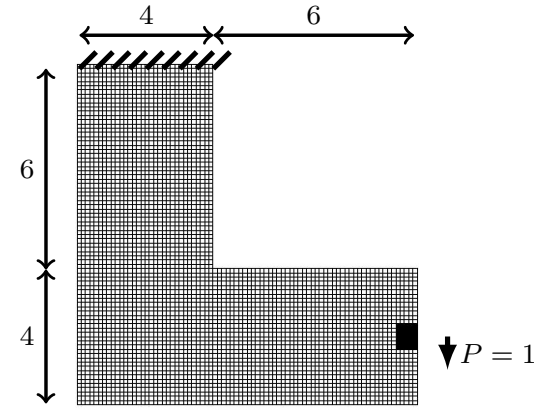

(b)

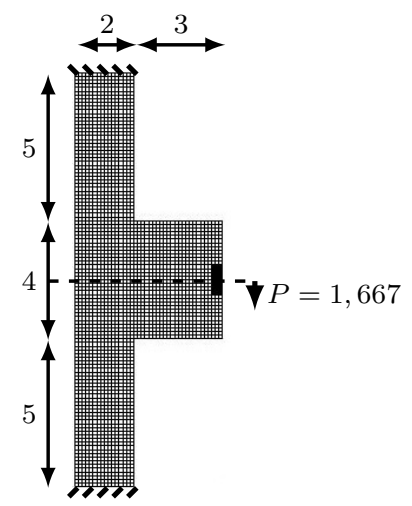

(c)

Fig. 3 Geometry and boundary conditions for the numerical applications (dimension in meters, forces in Newton for an unitary thickness). (a) cantilever long, (b) L-Shape lamina and (c) Half-H lamina

throughout this section. For fatigue design, additional material parameters are required in order to build the Goodman diagram in Figure 2. The yield stresses in tension/compression are assumed to be the same, i.e. $S_{y}=S_{y c}=5 \mathrm{~N} / \mathrm{m}^{2}$. The ratios $S_{e} / S_{y}$ and $S_{u t} / S_{y}$ are inspired by material AISI STEEL 1095, i.e. 0, 7195 and $=1,439$ respectively. If $S_{y}$ is assigned, the real proportions of the Goodman diagram for the considered material are preserved, as needed to provide full physical meaning of the simulations presented next.

Table 1 compares the achieved optimized layouts in terms of non-dimensional weight $\mathcal{W} / \mathcal{W}_{0}$ (the initial weight $\mathcal{W}_{0}$ is set to 100) and nondimensional compliance $\mathcal{C} / \mathcal{C}_{0}$, providing additional information on the adopted numerical settings (the number of active constraints at the final iteration $N_{a}^{f}$ and the CPU cost). Subscript o refers to the full domain made of virgin material, mean- 
ing that weight and compliance at convergence are normalized with respect to reference values at the first iteration of the optimization procedure, when $x_{e}=1, \forall e$.

Optimized layouts are presented in terms of the physical unknowns $\tilde{x}_{e}$ of Eqn. (12) and are endowed with maps representing the element-wise equivalent stress measure $\sigma_{G D \text { max }}^{e q}$ for fatigue-based layouts, or $\sigma_{V M, \max }^{e q}$ for strength-based results. For each example all the maps are normalized to get bounded within zero stress (blue regions) and the maximum equivalent stress (red regions).

The maximum l.h.s. of the sets of constraints in the Goodman criterion reads, see Eqn. (5):

$\sigma_{G D, \max }^{e q}=\max \left(\frac{\sigma_{a}^{e q}}{S_{e}}+\frac{\sigma_{m}^{e q}}{S_{u t}}, \frac{\sigma_{a}^{e q}}{S_{y}}-\frac{\sigma_{m}^{e q}}{S_{y c}}, \frac{\sigma_{a}^{e q}}{S_{e}}\right)$.

In fact, the Goodman criterion is enforced through three sets of inequalities and the value $\sigma_{G D, \max }^{e q}$ is the largest among the constraints prescribed by the criterion. The maximum l.h.s. of a conventional Von Mises constraint is given by:

$\sigma_{V M, \max }^{e q}=\frac{\sigma_{a}^{e q}}{S_{y}}$.

\subsection{Example 1. The long cantilever}

The first example deals with the optimization of a long cantilever which consists of a rectangular design domain where the left side is clamped and a load $P=0,834 N$ is applied downward as shown in Figure 3(a). Solving the problem through the strength-based optimization with compliance constraint (MWCS) leads to the design and the relevant stress map represented in Figure 4(a). Results achieved through the fatigue-constrained optimization proposed in this work are shown in Figures 4(b), 4(c) and 4(d). The enforced global compliance regularization parameter reads $\alpha_{c}=2$, meaning that the achieved optimal design has to preserve at least half of the original stiffness of the full material structure. The same assumption on $\alpha_{c}$ will be adopted for all the compliance-constrained numerical simulations presented in Section 4.

The MWCS solution presents full symmetry with respect to the horizontal axis, both in the design plot and in the relevant stress map. This is due to the adoption of the Von Mises strength criterion. Looking at Figure 4(a), small rounded corners appear in the vicinity of the clamped edges at the intersection of the trusses, aiming at of mitigating stress concentration in these regions.

Referring to the fatigue-constrained design, three cases are investigated here. The first one $\left(\mathrm{MWCF}_{1}\right)$ considers a large amount of alternating component $\left(c_{a}=0,7\right.$ and $\left.c_{m}=0,3\right)$, the second one $\left(\mathrm{MWCF}_{2}\right)$ includes as much alternating part as mean part $\left(c_{a}=0,5\right.$ and $\left.c_{m}=0,5\right)$, whereas the third one $\left(\mathrm{MWCF}_{3}\right)$ includes more mean part than alternating part $\left(c_{a}=0,3\right.$ and $\left.c_{m}=0,7\right)$. These combinations will be used also in Sections 4.2 and 4.3, preserving their acronyms.

The optimal design in Figure 4(b) has a topology that is very similar to the one represented in Figure 4(a), although the symmetry with respect to the horizontal axis is lost. This fact is due to the nature of the adopted fatigue criterion. Recalling Figure 2, it is clear that elements undergoing traction (first quadrant) are subject to stricter constraints than those in compression (second quadrant). Looking at Figure 4(b), a larger rounded corner appears at the connection between the horizontal tie and the inclined brace with respect to the MWCS design of Figure 4(a). This result is expected, because fatigue design essentially involves a reduction of any stress peak. Additionally, some structural members of the fatigue-constrained optimal layout are thicker than those of the strengthconstrained one. Indeed, the weight of the fatiguebased optimal design is larger, see Figure 5(a) and Table 1. The final compliance is twice its initial value, as requested, whereas Figure 6 shows that all the elements included in the design domain lie within the Goodman safety region. Figure 6 represents the stress coordinates $\left(\sigma_{m}^{e q}, \sigma_{a}^{e q}\right)$ evaluated at the centroid of all the elements in the domain, except those around the load application point (black regions in Figure 3(a)).

According to Eqn. (2), the component of the alternate and mean part of the stress $\sigma_{a, i j}$ and $\sigma_{m, i j}$ can be achieved scaling the reference stress $\sigma_{i j}$ by $c_{a}$ and $c_{m}$, respectively, being $c_{a}+c_{m}=1$. This means that a proportionality between these components exists, i.e.:

$\sigma_{a, i j}=\frac{c_{a}}{c_{m}} \sigma_{m, i j}$.

To move from the components $\sigma_{a, i j}$ and $\sigma_{m, i j}$ to the equivalent stress measures $\sigma_{m}^{e q}, \sigma_{a}^{e q}$ plotted in Figure 6, one has to apply Eqn. (3), involving linear and quadratic invariants. In case of elements that are subject to an uniaxial stress state $\bar{\sigma}$, this simply reads:

$\left\{\begin{array}{l}\sigma_{a}^{e q}=\frac{c_{a}}{c_{m}} \sigma_{m}^{e q}, \text { for } \bar{\sigma}>0 \\ \sigma_{a}^{e q}=-\frac{c_{a}}{c_{m}} \sigma_{m}^{e q}, \text { for } \bar{\sigma}<0\end{array}\right.$,

thus leading to the main $\mathrm{V}$-shape arising in the $\sigma_{m}^{e q}-\sigma_{a}^{e q}$ diagram. Elements subject to multi-axial 
stress states give rise to the remaining cloud of points.

A very limited number of points are located along the boundary of the fatigue criterion, corresponding to the few fully stressed elements with regard to the equivalent measure $\sigma_{G D \text {, max }}^{e q}$ in the map of Figure 4(b). These elements are crucial from a mechanical point of view, since their stress regime governs the fatigue-constrained design. A wider set of constraints control the numerical procedure since the active constraints selection strategy is not limited to the fully-stressed elements, see Section 3.3.

When a lower percentage of alternating component is considered within the optimization problem, the optimal layouts and stress maps illustrated in Figure 4(c) and 4(d) are found. The topology achieved for these examples is different than in the previous simulations. Two members are needed near the loading area instead of one, as it was the case both for the MWCS and for the $\mathrm{MWCF}_{1}$ problem. Assuming the weight of the MWCS design as a reference, a heavier structure arises for the topology in Figure 4(c), whereas nearly the same weight is found for the layout represented in Figure 4(d). Convergence of the objective function is reported in Figure 5(a), see also Table 1.

Concerning the computational performance, one can observe that convergence is slightly different when considering the MWCS, $\mathrm{MWCF}_{1}, \mathrm{MWCF}_{2}$ or $\mathrm{MWCF}_{3}$ problem. The number of iterations needed by each optimization run to achieve convergence is not the same, mainly because of the different set of active local constraints. As shown in Figure 5(b) and summarized in Table 1, the size of the active set grows with increasing the amount of alternating component. The influence on the overall CPU time is shown in Figure 5(c), where it can be seen that the $\mathrm{MWCF}_{1}$ problem takes more than four times the CPU effort of the MWCS problem. Moreover, the number of active constraints increases doing some jumps during the optimization process tackling the $\mathrm{MWCF}_{1}$ problem. This is a consequence of the adoption of an adaptive strategy for the relaxation parameter $q$, see Section 3.3. As expected, it may be concluded that the fatigueconstrained design requires an increased computational cost with respect to a conventional strengthconstrained optimization.

It must be remarked that a long stabilization plateau is obtained for each simulation in Figure 5(a). In order to ensure full convergence, optimization is stopped when the maximum difference of the

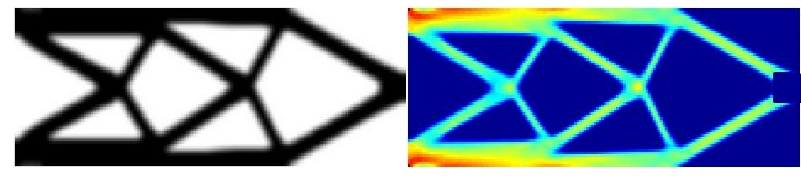

(a)

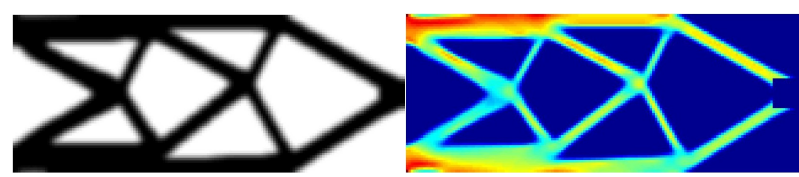

(b)

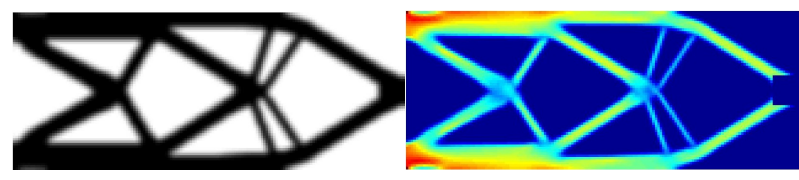

(c)

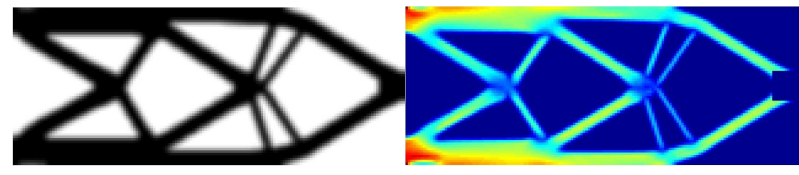

(d)

Fig. 4 Example 1. Optimized topologies and relevant stress maps for the MWCS and MWCF formulations of Example 1: (a) MWCS, (b) $\mathrm{MWCF}_{1}\left(c_{a}=0.7 ; c_{m}=0.3\right)$, (c) $\mathrm{MWCF}_{2}\left(c_{a}=0.5 ; c_{m}=0.5\right)$ and (d) $\operatorname{MWCF}_{3}\left(c_{a}=\right.$ $\left.0.3 ; c_{m}=0.7\right)$ )

value of each design variable between two steps is $\leq 0,005$. 


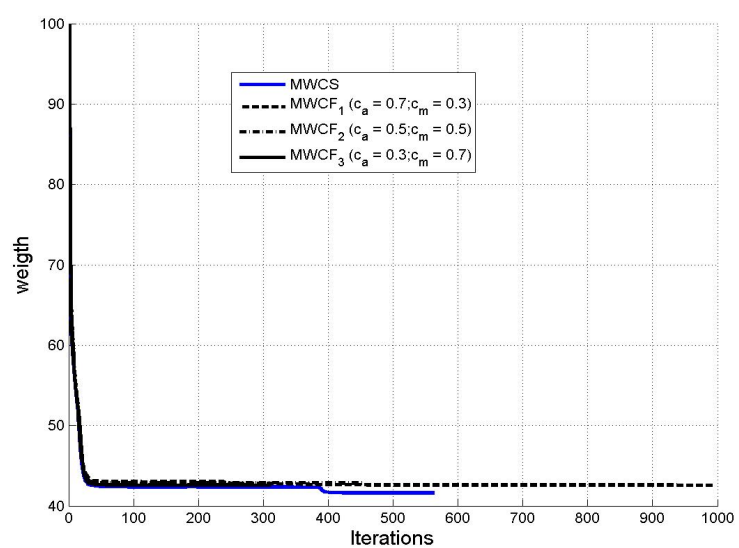

(a)

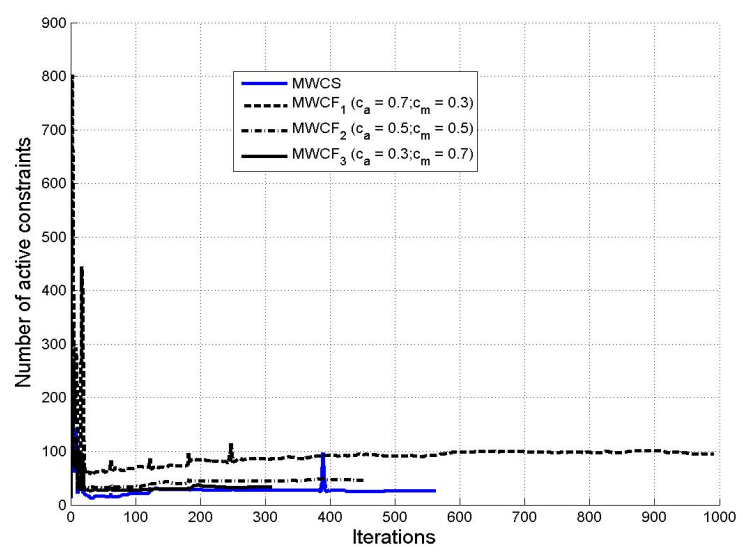

(b)

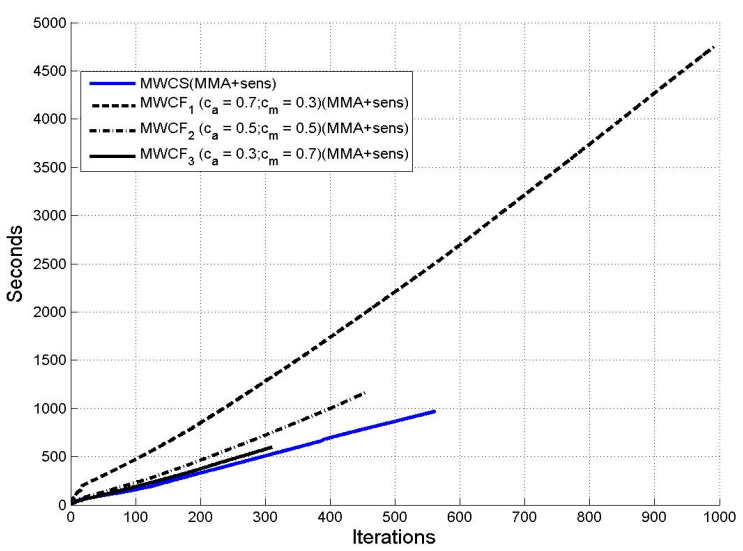

(c)

Fig. 5 Comparison of the evolution of the objective function (a), number of active constraints (b) and CPU requirements (c) for the three strategies of example 1

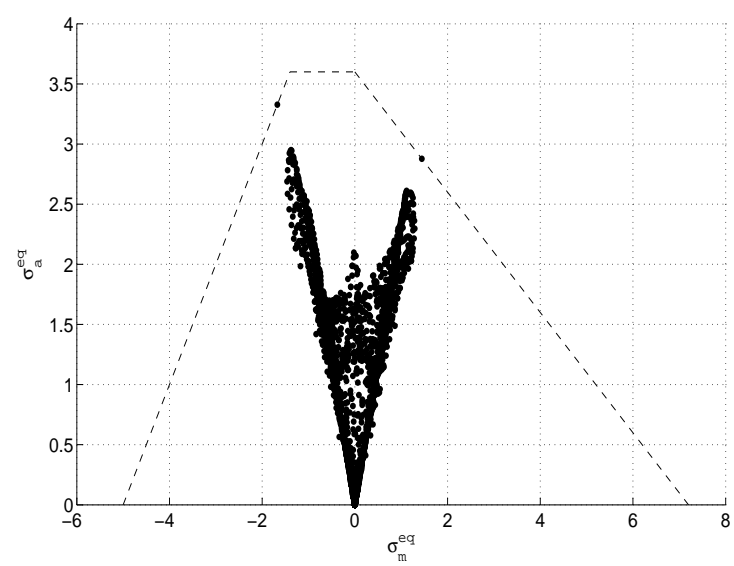

(a)

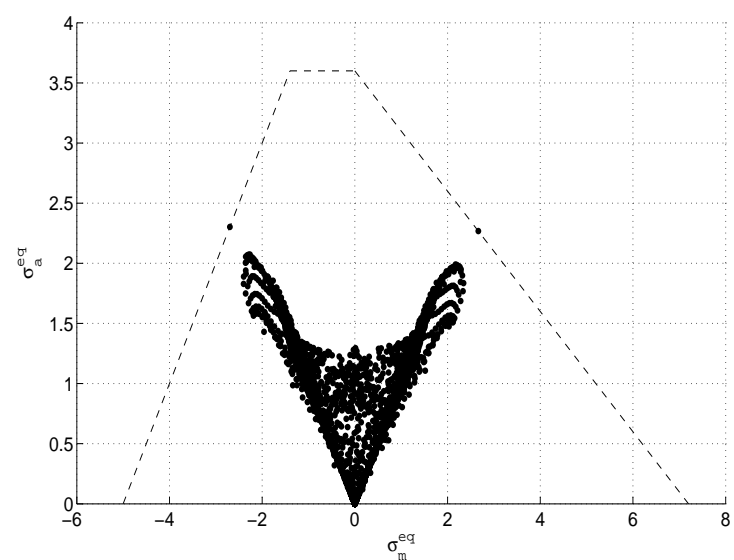

(b)

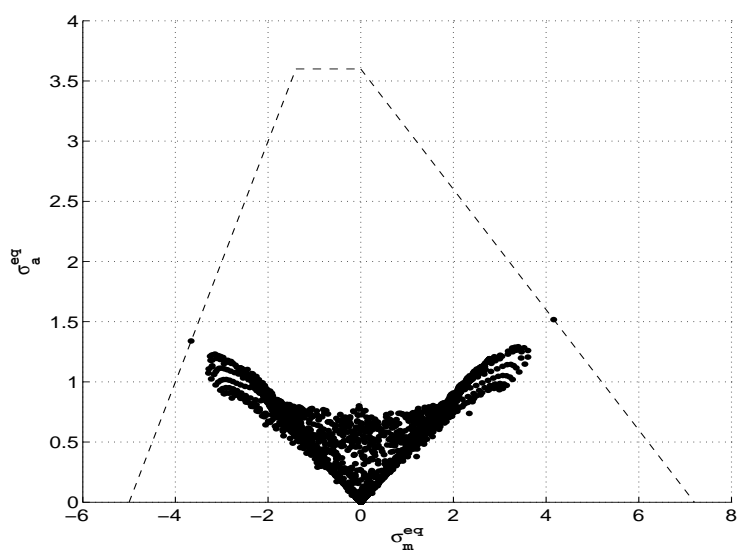

(c)

Fig. 6 Goodman diagram for the cantilever long with : (a) $c_{a}=0.7 ; c_{m}=0.3$, (b) $c_{a}=0.5 ; c_{m}=0.5$, (c) $c_{a}=$ $0.3 ; c_{m}=0.7$ 


\subsection{Example 2. The L-shaped plate}

The second example deals with the well-known Lshaped plate for which boundary conditions and loads $(P=1 N)$ are represented in Figure $3(\mathrm{~b})$. As in the previous section, the strength-constrained problem is assumed as the reference case, whereas problems of fatigue-based design are considered for the three combinations of weighting factors $c_{a}$ and $c_{m}$ introduced above.

The optimal design for the MWCS problem and its relevant stress map are shown in Figure 7(a). As extensively investigated in the literature, the achieved design is very different from a compliancebased layout since most of the material is removed from the corner to allow the stress path effectively circumvent the geometric singularity. A smoother design of the corner region could be achieved by means of the adoption of an advanced filtering approach, see Svärd (2015) and remarks in Section 3.3. The relevant stress map shows that maximum values of the equivalent stress arise in the vertical members that undergoes traction. Table 1 confirms that a suitable set of constraints is active at convergence to fulfill the prescribed Von Mises strength criterion and meet the stiffness requirement.

Remarks can be formulated looking at the optimal design achieved for the $\mathrm{MWCF}_{1}$ problem. Although the optimal layout seems quiet similar to the assumed reference MWCS, some noticeable differences arise. A three-member sub-structure appears in the fatigue-constrained layout of Figure $7(\mathrm{~b})$, replacing the two-member sub-structure that connects tensile-stressed and compressive -stressed paths in the bulk of the domain. It also appears that the optimization enlarges the members around the corner and rounds the design nearby, with an impact on the total weight of the structure, see Figure 8(a). Indeed, the pronounced smoothing of any geometric singularity is a peculiar feature of fatigue-constrained design, which aims at reducing any localized stress peak to prevent crack initiation.

Moreover, the stress map referring to the $\mathrm{MWCF}_{1}$ design allows pointing out that a wider region of the structure reaches the maximum equivalent values that are allowed by the adopted fatigue criterion. This is the case of the thick vertical tie connecting the clamped edge to the corner. Figure 9(a) confirms that all the discrete elements that approximate the optimized design are safely stressed with respect to the Goodman criterion, whereas Table 1 indicates that the expected stiff- ness is reached. As usual, some design points lie along the boundary of the fatigue criterion, meaning that the relevant elements need special care (their safety factor is unitary). Figure 8(b) shows that the number of retained active constraints is more than four times the number of active constraints of the strength-constrained reference design. This has a clear impact on the overall CPU time, as pointed out in Table 1 and Figure 8(c).

Further investigations are reported involving the $\mathrm{MWCF}_{2}$ problem and the $\mathrm{MWCF}_{3}$ problem, with results shown in Figure $7(\mathrm{c})$ and $7(\mathrm{~d})$, respectively. Lighter structures arise in both cases, as summarized in Table 1, see also Figure 8(a). The thickness of the trusses is reduced, whereas the curvature around the sharp corner increases, suggesting that the amount of the alternate part of the stress with respect to the mean part plays a crucial role in the fatigue-based design of the $\mathrm{L}_{-}$ shaped cantilever. Although the optimized design achieved for the $\mathrm{MWCF}_{2}$ problem is very similar to that found for the reference MWCS problem, an additional structural member arises in the fatigueconstrained design. From Figure 7 (c), it can bee noticed that stress is low in this thin member, meaning that it could be easily erased by increasing the filtering radius. Once again, the number of active constraints is bigger than in the reference case, leading to an increased computational burden, see Figure 8(c).

The solution of the $\mathrm{MWCF}_{3}$ problem is almost the same achieved for the reference strength-constrained solution. This result can be explained looking at the $\mathrm{V}$-shaped cloud of points shown in Figure 9(c)). Indeed, decreasing the amount of alternate component means moving the cloud towards the lower part of the diagram, where the feasible region is larger and higher values of the stress are allowed, being not far from $S_{y}$.

A problem implementing only fatigue constraints has been introduced to investigate the L-shaped cantilever when dropping the compliance enforcement. The case with $c_{a}=0.7$ and $c_{m}=0.3$ has been chosen to compare the result achieved through a pure fatigue-based minimization of the weight (MWF 1 problem) with respect to the multi-constrained $\mathrm{MWCF}_{1}$ problem seen before. The same selection strategy is applied in both cases.

The achieved layouts are represented in Figure 10, whereas Figure 11 provides a history plot of the objective function and of the CPU time. Due to the adoption of the same fatigue criterion, the optimal structures are very similar. Con- 
versely, smoothness of the objective function becomes rather bad when dropping the compliance constraint and the CPU time remarkably increases. Of course, one could enlarge the set of active constraints to be passed to the minimizer. This would mitigate convergence instabilities, but would call for an extra-cost in terms of CPU time.

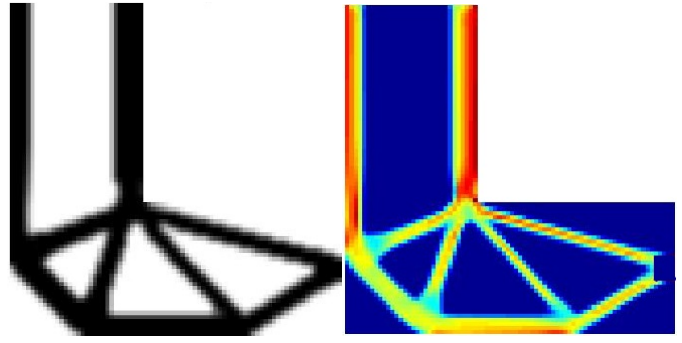

(a)

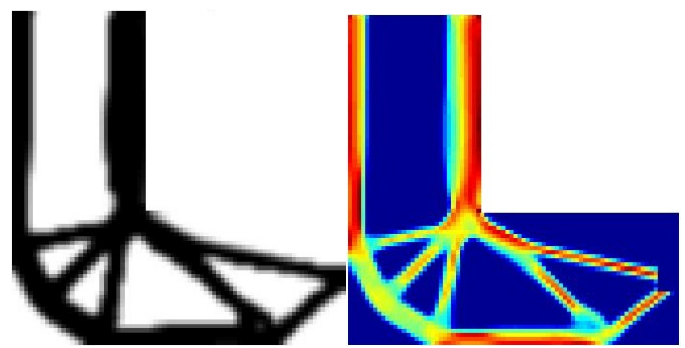

(b)

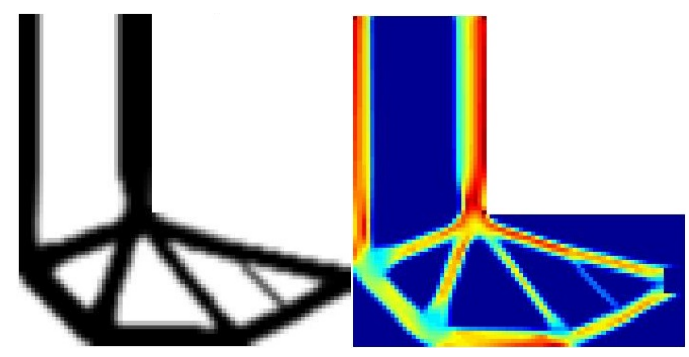

(c)

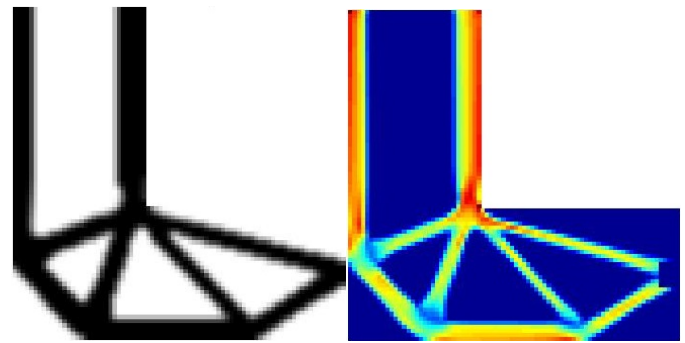

(d)

Fig. 7 Example 2. Optimized topologies and relevant stress maps for the MWCS and MWCF formulations of Example 2: (a) MWCS, (b) $\mathrm{MWCF}_{1}\left(c_{a}=0.7 ; c_{m}=0.3\right)$, (c) $\mathrm{MWCF}_{2}\left(c_{a}=0.5 ; c_{m}=0.5\right)$ and (d) $\mathrm{MWCF}_{3}\left(c_{a}=\right.$ $\left.0.3 ; c_{m}=0.7\right)$ ) 


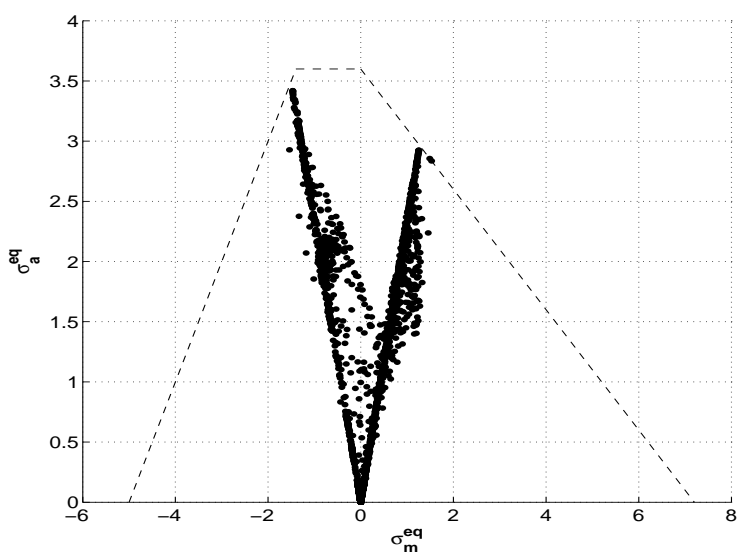

(a)

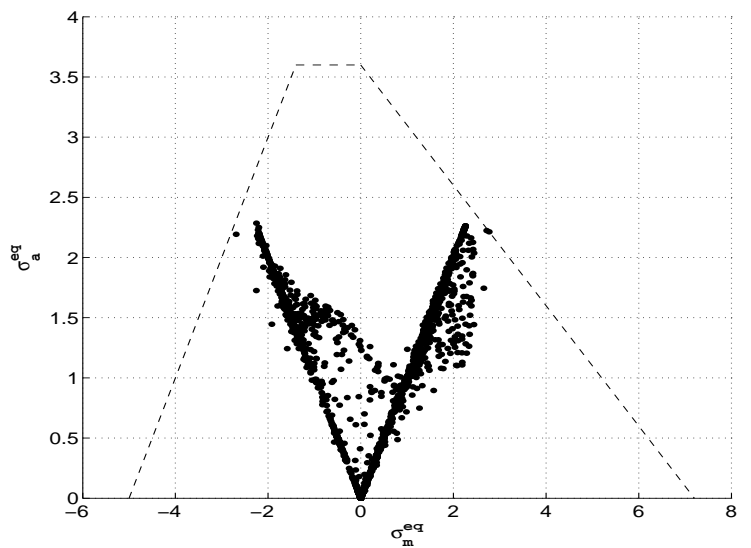

(b)

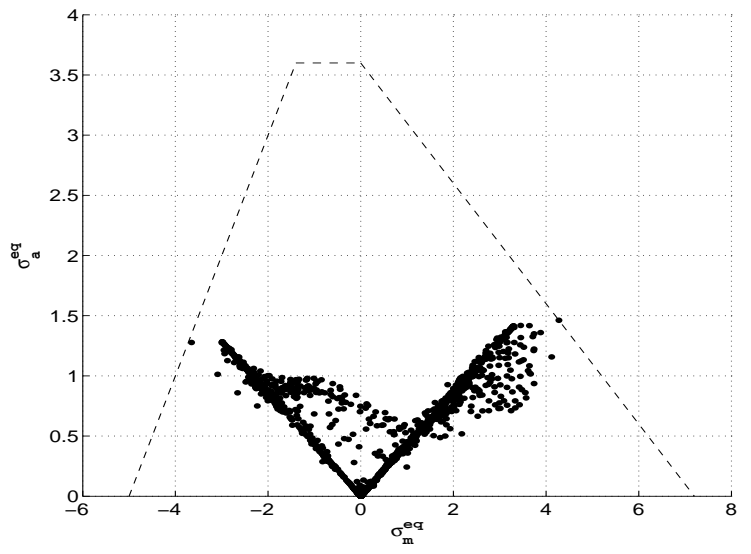

(c)

labelfig:Lgall

Fig. 9 Goodman diagram for the $\mathrm{L}-$ Shape lamina with : (a) $c_{a}=0.7 ; c_{m}=0.3$, (b) $c_{a}=0.5 ; c_{m}=0.5$, (c) $c_{a}=$ $0.3 ; c_{m}=0.7$

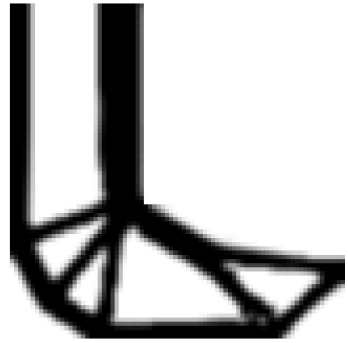

(a)

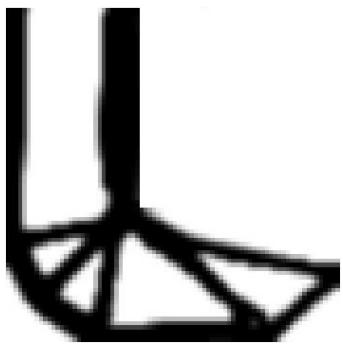

(b)
Fig. 10 Comparison of $\mathrm{MWF}_{1}$ and $\mathrm{MWCF}_{1}$ problems: a) $c_{a}=0,7$ and $\left.c_{m}=0,3 \mathrm{~b}\right) c_{a}=0,7$ and $c_{m}=0,3$ and $\alpha_{c}=2$

\subsection{Example 3. The half-H lamina}

For this third example, the design domain of Figure $3(\mathrm{c})$ is considered, loaded by a vertical force $P=1,667 N$. Once again, the solution of the strengthconstrained MWCS problem and the three versions of the fatigue-constrained problem are investigated. The relevant layouts and stress maps are given in Figure 14(a) for the reference solution and in Figures 14(b), 14(c) and 14(d) for the $\mathrm{MWCF}_{1}$, $\mathrm{MWCF}_{2}$ and $\mathrm{MWCF}_{3}$ problems, respectively.

This last example confirms conclusion from the two previous benchmarks. The optimal layouts found by the considered optimization problems are along the lines of the previous results: the members of the structures achieved through fatigue-based procedures are thicker than those of the reference one, leading to heavier structures, see Figure 15(a) and Table 1. Furthermore, looking at Figures 16(a) to 16(c), one can see that all the elements used to approximate the design domain meet the expectations regarding fatigue resistance, with most of the points showing a safety factor much bigger than unity. As seen for the L-shaped specimen of Section 4.2, the fatigue-constrained design becomes very similar to the strength-constrained layout when the mean component prevails on the alternate component of the stress. As outlined for the long cantilever of Section 4.1, slightly nonsymmetric layouts are expected in case of fatigueconstrained optimization, due to the different shape of the adopted Goodman diagram in the first quadrant $\sigma_{m}^{e q}>0$ and in the second one $\sigma_{m}^{e q}<0$.

This example also confirms that the computational cost for problems involving fatigue design is generally higher with respect to the strengthbased framework. Indeed, the optimization has to 
handle an increased number of constraints, see Figure 15(b) and especially the $\mathrm{MWCF}_{1}$ problem. When the alternate component of the stress prevails on the mean one, the CPU time noticeably increases with respect to a strength-based problem enforcing the symmetric von Mises criterion. This is consistent with physical expectation, suggesting that the alternating part is the most critical one.

\section{Conclusions}

Fatigue is a complex mode of failure that needs to be considered in the early stage of the design of any mechanical component to select appropriate topology. However, in case of high number of cycles, dynamic analysis can be replaced by a static one and fatigue resistance can be preliminary enforced through an intuitive design rule that consists in assessing the feasibility of the stress regime throughout the domain with respect to a prescribed criterion.

A simplified approach has been proposed herein to cope with the minimization of the weight of linear elastic structures under local fatigue constraints and a global compliance enforcement. The expected stiffness of the optimized design is provided by the global constraint, whereas a set of local stress-based enforces the structural fatigue resistance. The Sines method has been used to define the equivalent mean and alternate stress in case of $2 \mathrm{D}$ and $3 \mathrm{D}$ problem depending on the invariants of the stress tensor and its deviatoric part, respectively. Hence, a modified Goodman fatigue criterion has been implemented through the same formalism needed to address compression-dependent failure in materials following the Drucker-Prager strength criterion, see Bruggi and Duysinx (2012). The so-called singularity phenomenon is overcome by the implementation of a suitable relaxation of the equivalent stress measures related to fatigue resistance.

As expected, fatigue-constrained optimal layouts circumvent geometric singularities to avoid the arising of any stress peak. Numerical examples show that fatigue design generally calls for heavier structures with respect to strength-based optimization for the von Mises criterion, thickening suitable parts of the optimal layout. When the alternating component of the stress prevails on the mean one, the achieved optimal truss-like structures are quite different with respect to conventional strength-based layouts, as explained by the shape of the Goodman diagram. Also, the adopted fatigue failure criterion is not symmetric with respect to the sign of the mean value of the stress, thus often leading to some minor asymmetry in the optimal structure.

Referring to computational issues, it has been found that the fatigue-constrained optimization is more demanding than the strength-constrained design. Indeed, the set of active local constraints steering the optimizer towards the achievement of stiff fatigue-resistant layouts strongly depends on the amount of alternating and mean component of the stress.

It must be remarked that the proposed approach is a simplified design tool that can improve conventional compliance-based layouts with respect to fatigue adopting a straightforward extension of available formulations for stress-constrained topology optimization. Notwithstanding the limited accuracy affecting the evaluation of the stress field within a topology optimization problem, the achieved layouts can be usefully exploited by the designer to get a preliminary insight and to initialize more accurate procedures of optimization and analysis based e.g. on shape optimization, as outlined in Section 1.

Acknowledgements Part of the work has been done when the first author was spending a research period at Politecnico di Milano. This author would like to acknowledge the Belgian National Fund for Scientific research (FRIA) for its financial support.

\section{Bibliography}

Andreassen E, Clausen A, Schevenels M, Lazarov BS, Sigmund O (2011) Efficient topology optimization in MATLAB using 88 lines of code. Struct Multidiscip Optim 43:1-16

Bendsøe M, Kikuchi N (1988) Generating optimal topologies in structural design using a homogeneization method. Comp Meth Appl Mech Eng 71:197-224

Bendsøe M, Sigmund O (2003) Topology optimization Theory, methods and applications, Springer, EUA, New York

Bourdin B (2001) Filters in topology optimization. Int J Numer Methods Eng 50:2143-2158

Bruggi M (2008) On an alternative approach to stress constraints relaxation in topology optimization. Struct Multidiscip Optim 36:125-141

Bruggi M, Dusyinx P (2012) Topology optimization for minimum weight with compliance and stress constraints. Struct Multidisc Optim 46(3):369-384

Bruggi M, Dusyinx P (2013) A stress-based approach to the optimal design of structures with unilateral behavior of material or supports . Struct Multidisc Optim 46(3):369-384

Budynas R.G, Nisbett J.K (2011) Shigley's Mechanical Engineering Design, 9th edition New York: McGraw-Hill 
Bruns TE, Tortorelli DA (2001) Topology optimization of non-linear elastic structures and compliant mechanisms. Comp Meth Appl Mech Eng 190:3443-3459

Cheng GD, Guo X (1997) $\varepsilon$-relaxed approach in topology optimization. Struct Optim 13: 258-266

Duysinx P, Bendsøe MP (1998) Topology optimization of continuum structures with local stress constraints. Int J Numer Methods Eng 43: 1453-1478

Duysinx P (1999) Topology optimization with different stress limits in tension and compression, In:Proceedings of the 3rd World Congress of Structural and Multidisciplinary Optimization WCSMO3

Duysinx P, Sigmund O (1998) New developments in handling stress constraints in optimal material distribution. 7th Symposium on Multidisciplinary Analysis and Optimization AIAA-98-4906: 1501-1509

Duysinx P, Van Miegroet L, Lemaire E, Brüls O, Bruyneel M (2008) Topology and generalized shape optimization: why stress constraints are so importants ? Int J.Simul.Multidisc.Des.Optim. 2:253-258

Eschenauer HA, Olhoff N (2001) Topology optimization of continuum structures: A review. Appl Mech Rev $54: 331-389$.

Grunwald J, Schnack E(1997) A fatigue model for shape optimization. Structural Optimization 14:36-44

Guilherme CEM, Fonseca JSO (2007) Topology optimization of continuum structures with epsilon-relaxed stress constraints. In:Alves M, da Costa Mattos HS (eds) Solid mechanics in Brazil, vol 1. ABCM, Rio de Janeiro, pp 239-250

Holmberg E, Torstenfelt B, Klarbring A (2013) Stress constrained topology optimization. Struc Multidisc Optim 48:33- 47

Holmberg E, Torstenfelt B, Klarbring A (2014) Fatigue constrained topology optimization. Struc Multidisc Optim 50:207-219

Kaya N, Karen I, Öztürk F (2010) Re-design of a failed clutch fork using topology and shape optimization by the response surface method. Materials and Design 31:3008-3014

Kim Y.I, Park G.J (2010) Nonlinear dynamic response structural optimization using equivalent static loads. Comput. Methods Appl. Mech. Engrg. 199:660-676

Kirsch U (1990) On singular topologies in optimal structural design. Struct Optim 2:133-142

C. Lalanne (1999) Mechanical vibration \& shock. Volume IV, Fatigue damage. Hermes Penton Science, 1999.19

Le C, Norato J, Bruns TE, Ha C and Tortorelli DA (2010) Stress-based Topology Optimization for Continua. Struct Multidiscip Optim 41:605-620

Luo Y, Yu Wang Yu M, Kang Z (2013) An enhanced aggregation method for topology optimization with local stress constraints. Comput. Method Appl. Mech. Engrg. 254:31- 41

Mrzygold M, Zielinski A.P 2006) Numerical implementation of multiaxial high-cycle fatigue criterion to structural optimization. Journal of Theoretical and Applied Mechanics, 44(3),pp 691-712

Norton R. L (2000). Machine design: An integrated approach. Upper Saddle River, N.J: Prentice Hall

París J, Navarrina F, Colominas I, Casteleiro M (2009) Topology optimization of continuum structures with local and global stress constraints. Struct Multidisc Optim 39(4):419-437

Park G.J, Kang B.S (2003)Validation of a structural optimisation algorithm transforming dynamic loads into equivalent static loads. Journal of optimization theory and applications: Vol 118, No.1., pp.191-200

Pereira JT, Fancello EA, Barcellos CS (2004) Topology optimization of continuum structures with material failure constraints. Struct Multidisc Optim 26(1-2):50-66

Rozvany GIN (2009) A critical review of established methods of structural topology optimization. Struct Multidiscip Optim 37:217-237

Seung H.J, Dong-Hoon C, Yoon G.H (2015) Fatigue and static failure considerations using topology optimization method. Applied Mathematical Modeling 39:1137 $-1162$

Sherif K. and Irschik H. (2010) Efficient Topology Optimization of Large Dynamic Finite Element Systems Using Fatigue. AIAA Journal vol.48, No.7, pp 1339-1347

Schütz W. (1996) A history of fatigue Engineering Fracture Mechanics Vol. 54, No. 2, pp. 263-300

Sigmund O (2007) Morphology-based black and white filters for topology optimization. Structural and Multidisciplinary Optimization 33(4-5):401-424

Sigmund O, Petersson J (1998) Numerical instabilities in topology optimization: a survey on procedures dealing with checkerboards, mesh-dependencies and local minima. Struct Optim 16:68-75

Svanberg K (1987) Method of moving asymptotes - A new method for structural optimization. Int J Numer Methods Eng 24:359-373

Svanberg K, Werme M (2007) Sequential integer programming methods for stress constrained topology optimization. Struct Multidisc Optim 34(4):277-299

Svärd H (2015) Topology Optimization of FatigueConstrained Structures, Doctoral Thesis, KTH Engineering Sciences, Stockholm, Sweden

Svärd H.(2015) Interior value extrapolation: a new method for stress, evaluation during topology optimization, Struct Multidisc Optim 51:613-629

Sved G, Ginos Z (1968) Structural optimization under multiple loading. Int J Mech Sci 10:803-805

Yang RJ, Chen CJ (1996) Stress-based topology optimization. Struct Multidisc Optim 12(2):98-105

Weibull W. A statistical theory of the strength of materials. Ingeniörsvetenskapsakademiens handlingar. Generalstabens litografiska anstalts förlag, 1939. 


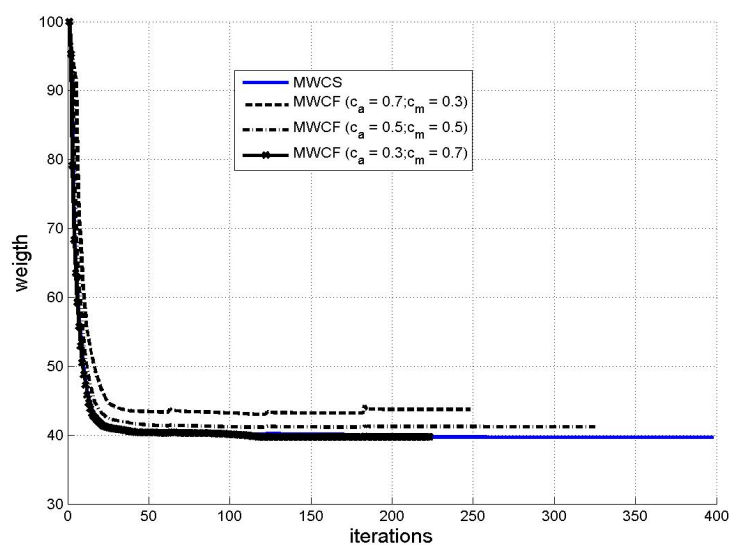

(a)

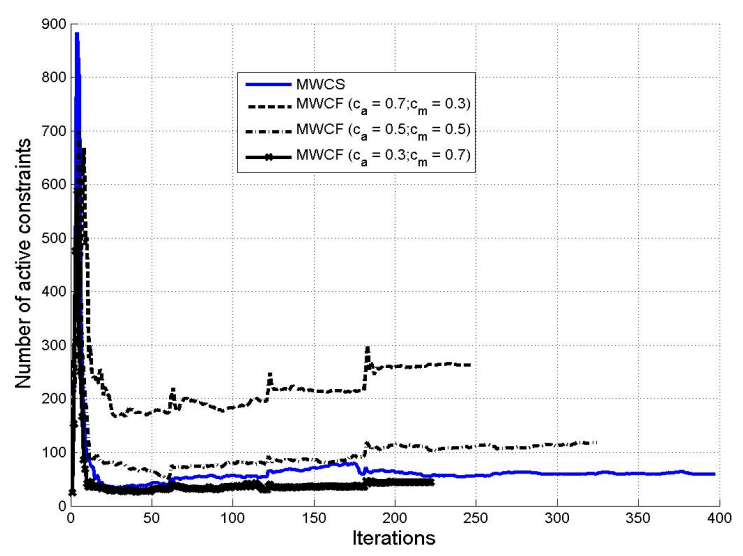

(b)

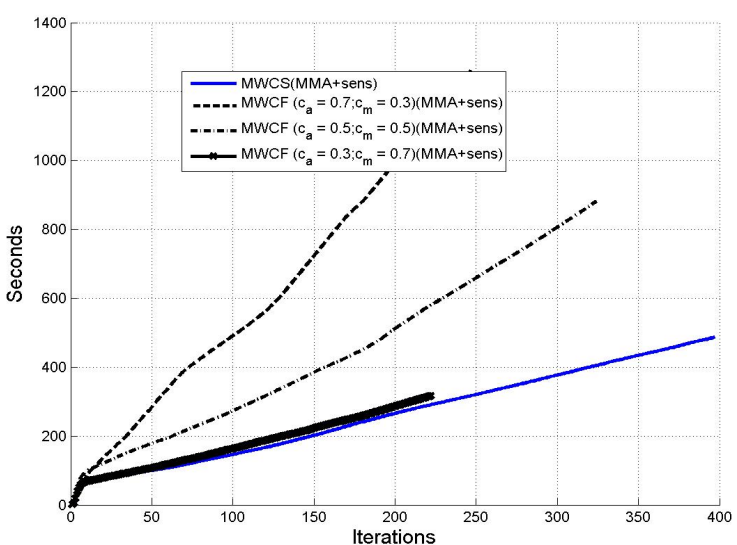

(c)

Fig. 8 Comparison of the evolution of the objective function (a), the number of active constraints (b) and the CPU requirement (c) for the three strategies of example 2 


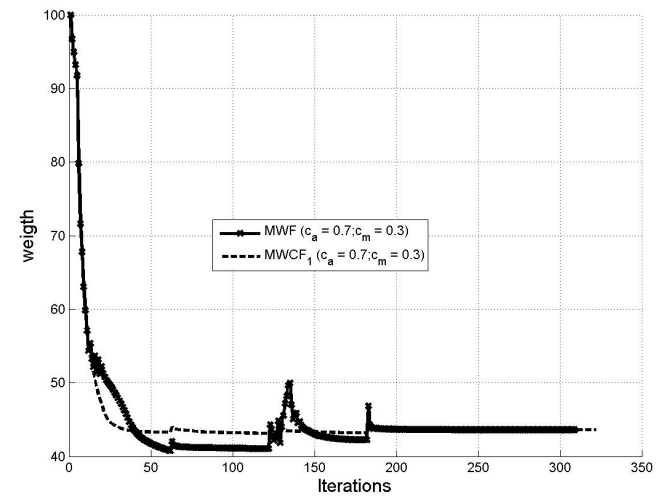

(a)

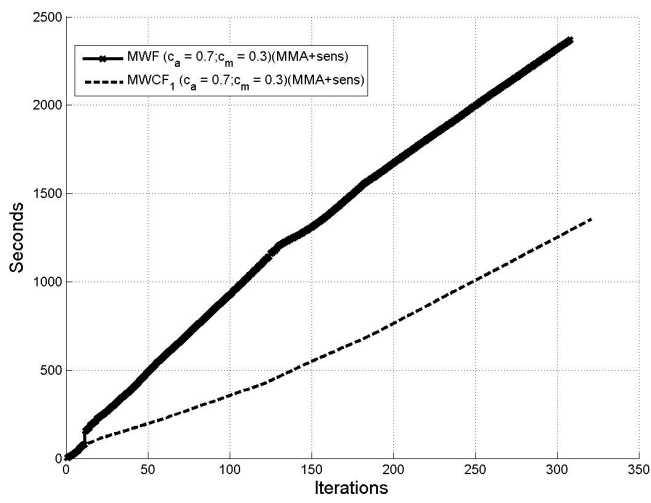

(b)

Fig. 11 Comparison of $\mathrm{MWF}_{1}$ and $\mathrm{MWCF}_{1}$ problems: a) Objective function b) CPU times

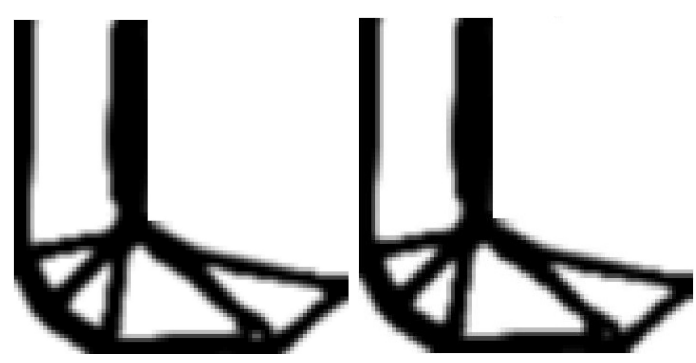

(a) $\left(^{*}\right)$

(b)

Fig. 12 Comparison of $\mathrm{MWCF}_{1}$ for real and fictious material properties: a) $c_{a}=0,7$ and $c_{m}=0,3\left({ }^{*}\right.$ stands for real material) b) $c_{a}=0,7$ and $c_{m}=0,3$ and $\alpha_{c}=2$ 


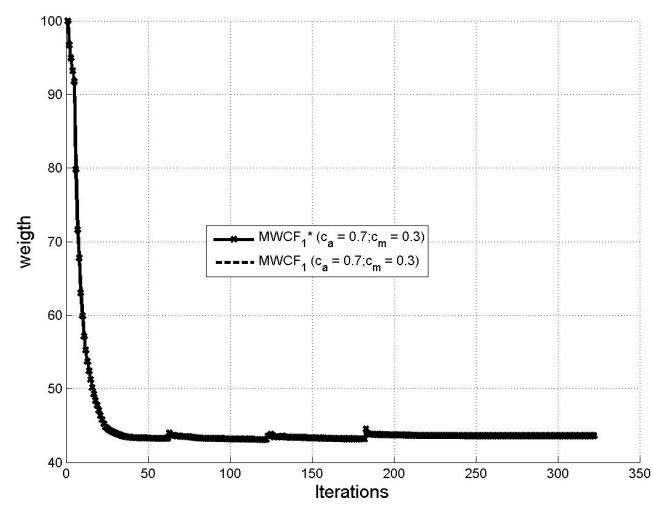

(a)

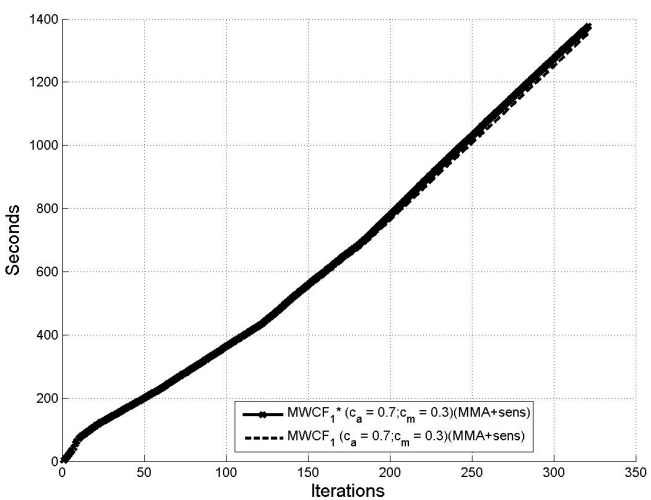

(b)

Fig. 13 Comparison of $\mathrm{MWCF}_{1}$ for real and fictious material properties ( ${ }^{*}$ stands for real material) : a) Objective function b) CPU times 


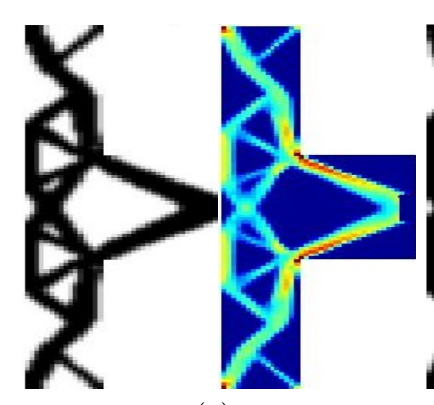

(a)

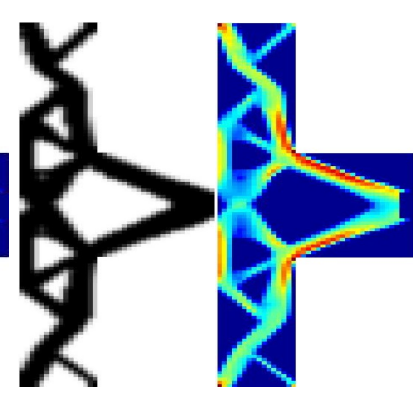

(b)

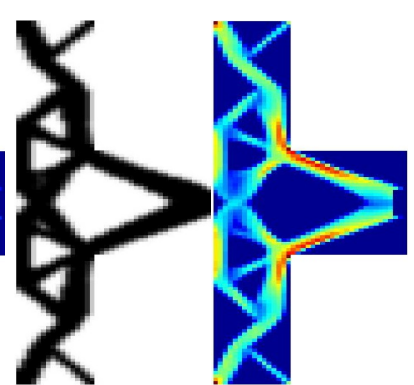

(c)

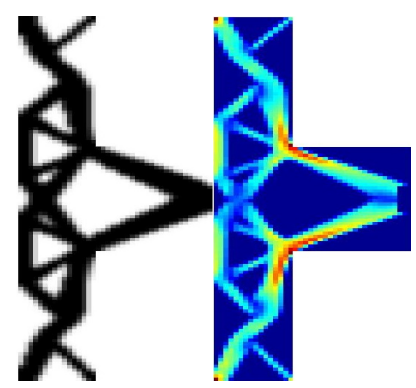

(d)

Fig. 14 Example 3. Optimized topologies and relevant stress maps for the MWCS and MWCF formulations of Example 3: (a) MWCS, (b) $\operatorname{MWCF}_{1}\left(c_{a}=0.7 ; c_{m}=0.3\right),(\mathrm{c}) \mathrm{MWCF}_{2}\left(c_{a}=0.5 ; c_{m}=0.5\right)$ and (d) $\left.\mathrm{MWCF}_{3}\left(c_{a}=0.3 ; c_{m}=0.7\right)\right)$ 


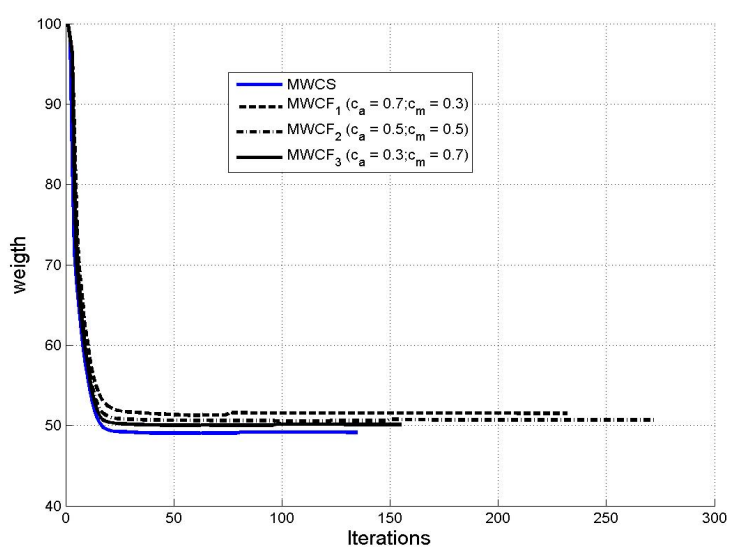

(a)

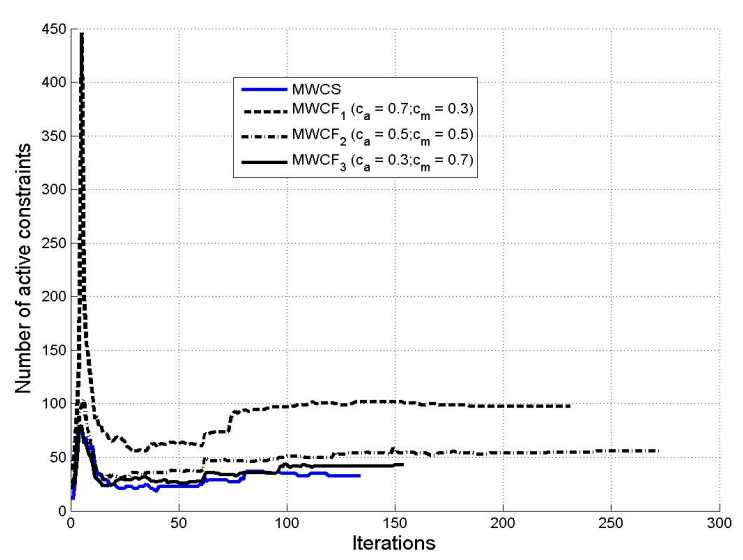

(b)

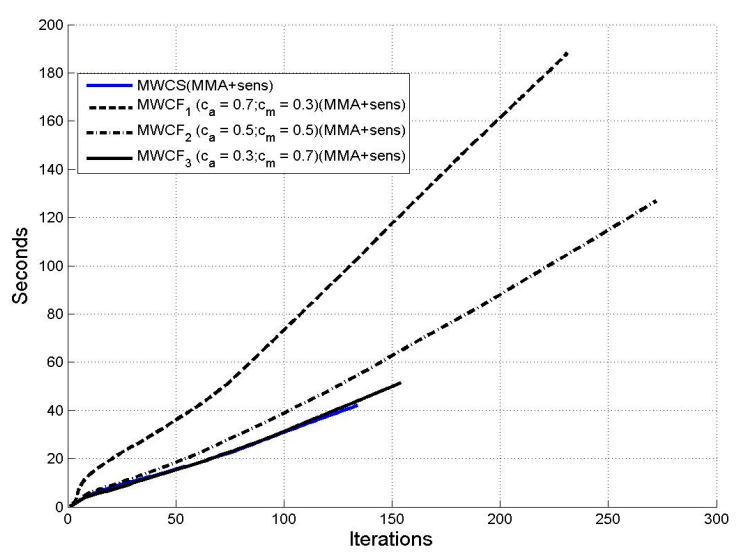

(c)

Fig. 15 Comparison of the evolution of the objective function (a), the number of active constraints (b) and the CPU requirement (c) for the three strategies of example 3

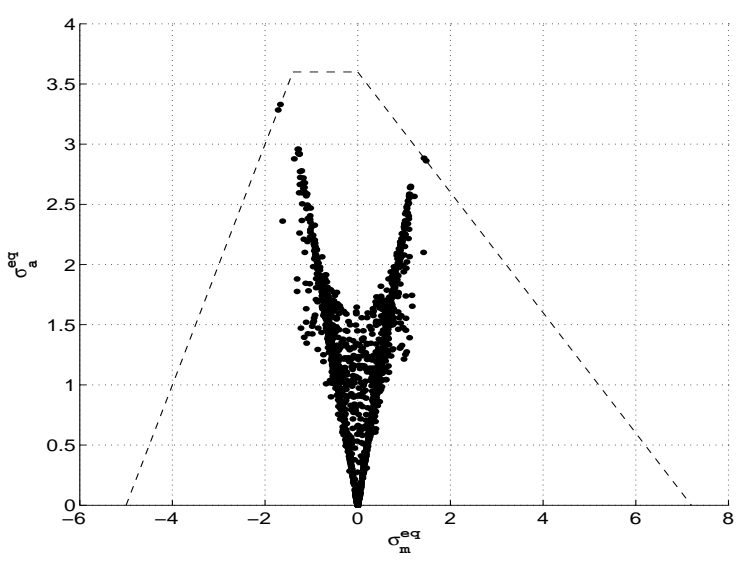

(a)

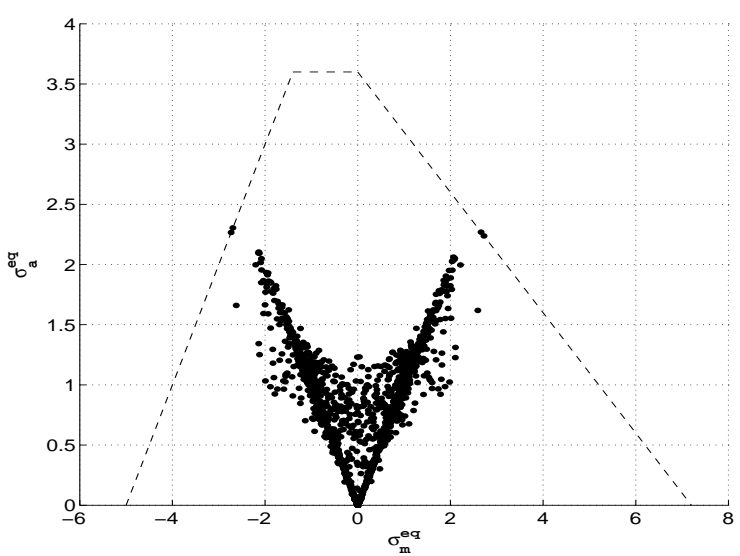

(b)

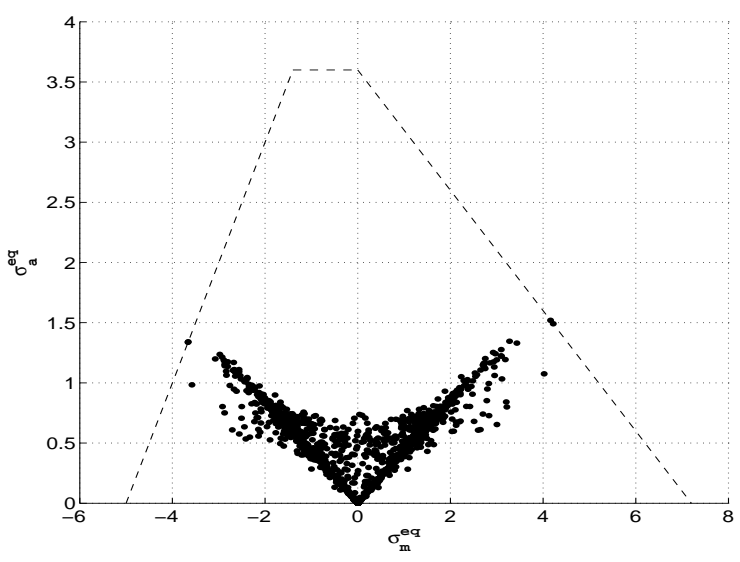

(c)

Fig. 16 Goodman diagram for the Half-H lamina with : (a) $c_{a}=0.7 ; c_{m}=0.3$, (b) $c_{a}=0.5 ; c_{m}=0.5$, (c) $c_{a}=$ $0.3 ; c_{m}=0.7$ 